\title{
Origin of plasma-induced surface roughening and ripple formation during plasma etching: The crucial role of ion reflection
}

\section{AUTHOR(S):}

Hatsuse, Takumi; Nakazaki, Nobuya; Tsuda, Hirotaka; Takao, Yoshinori; Eriguchi, Koji; Ono, Kouichi

\section{CITATION:}

Hatsuse, Takumi ...[et al]. Origin of plasma-induced surface roughening and ripple formation during plasma etching: The crucial role of ion reflection. Journal of Applied Physics 2018, 124(14): 143301.

\section{ISSUE DATE:}

2018-10-14

URL:

http://hdl.handle.net/2433/234709

\section{RIGHT:}

The following article appeared in 'Journal of Applied Physics 124, 143301 (2018)' and may be found at

https://aip.scitation.org/doi/10.1063/1.5041846.; The full-text file will be made open to the public on 14 October 2019 in accordance with publisher's 'Terms and Conditions for Self-Archiving' 


\title{
Origin of plasma-induced surface roughening and ripple formation during plasma etching: The crucial role of ion reflection
}

\author{
Takumi Hatsuse, ${ }^{1, a)}$ Nobuya Nakazaki, ${ }^{1, b)}$ Hirotaka Tsuda, ${ }^{1, c)}$ Yoshinori Takao, ${ }^{1, d)}$ \\ Koji Eriguchi, ${ }^{1}$ and Kouichi Ono ${ }^{1,2, e)}$ \\ ${ }^{1}$ Department of Aeronautics and Astronautics, Graduate School of Engineering, Kyoto University, \\ Kyoto-daigaku Katsura, Nishikyo-ku, Kyoto 615-8540, Japan \\ ${ }^{2}$ Joining and Welding Research Institute, Osaka University, 11-1 Mihogaoka, Ibaraki, Osaka 567-0047, Japan
}

(Received 27 May 2018; accepted 15 September 2018; published online 11 October 2018)

\begin{abstract}
Plasma-induced surface roughening and ripple formation has been studied based on Monte Carlo simulations of plasma-surface interactions and feature profile evolution during $\mathrm{Si}$ etching in $\mathrm{Cl}-$ based plasmas, with emphasis being placed on the role and effects of ion reflection from microstructural feature surfaces on incidence. The simulation model included the effects $\mathrm{of}^{+} \mathrm{Cl}^{+}$ion reflection (and/or its penetration into substrates) through calculating the momentum and energy conservation in successive two-body elastic collisions with substrate Si atoms every ion incidence. The "reflection coefficient $r_{i}$ " was then further introduced in the model $\left(0 \leq r_{i} \leq 1\right)$, representing the fraction of ions incident on surfaces with the reflection/penetration calculation scheme turned on. The coefficient $r_{i}$ is, in a sense, a measure of the reflection probability for impacts of an ion species onto Si surfaces relative to that for $\mathrm{Cl}^{+}$impacts. Simulations for ion incidence angles of $\theta_{i}=0^{\circ}, 45^{\circ}$, and $75^{\circ}$ onto substrate surfaces with incident energies in the range $E_{i}=20-500 \mathrm{eV}$ showed that as $r_{i}$ is slightly decreased from unity, the roughness decreases substantially, and the ripple formation fades away: the roughness remains at the low level of stochastic roughening during etching for decreased $r_{i} \leq$ $r_{i}^{*} \approx 0.95-0.75$ (the critical $r_{i}^{*}$ tends to be lower at higher $E_{i}$ and $\theta_{i}$ ) with no ripple structures at off-normal $\theta_{i}$. This elucidates that the ion reflection is indispensable in surface roughening and rippling during plasma etching, and their degree relies significantly on the reflectivity of ions. Simulations further showed that at intermediate off-normal $\theta_{i}=45^{\circ}$, the ripple wavelength increases significantly with decreasing $r_{i}$, while the increase in amplitude is relatively less significant; thus, sawtooth-like ripple profiles pronounced for $r_{i}=1$ tend to be collapsed with decreasing $r_{i}$. These effects of reduced ion reflection on plasma-induced surface roughening and ripple formation are discussed in terms of effectively enhanced smoothing due to neutral reactants, which competes with the roughening and rippling caused by ion bombardment. Published by AIP Publishing.

https://doi.org/10.1063/1.5041846
\end{abstract}

\section{INTRODUCTION}

Plasma-induced surface roughness at atomistic scale has become an important issue in the fabrication of nanoscale microelectronic and microelectromechanical devices, ${ }^{1,2}$ because it largely affects the variability in device performance. ${ }^{3,4}$ Surface roughening during plasma etching has been studied extensively, ${ }^{5-7}$ including sidewall roughening in pattern transfer ${ }^{8-15}$ and also maskless formation of organized nanostructures such as nanotextures and nanopillars. ${ }^{16-21}$ Correspondingly, several mechanisms have been invoked in

\footnotetext{
a)Present address: Nippon Steel \& Sumitomo Metal Corporation, Nagoya Works, Tokai, Aichi 476-8686, Japan.

${ }^{b)}$ Present address: Sony Semiconductor Solutions Corporation, Device Development Division, Atsugi, Kanagawa 243-0014, Japan.

${ }^{c)}$ Present address: Toshiba Corporation Semiconductor \& Storage Products Company, Center for Semiconductor Research \& Development, Kawasaki, Kanagawa 212-8583, Japan.

d) Present address: Division of Systems Research, Faculty of Engineering, Yokohama National University, Yokohama, Kanagawa 240-8501, Japan.

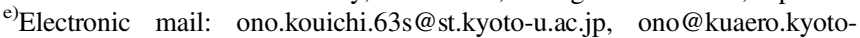
u.ac.jp, and ono@jwri.osaka-u.ac.jp
}

continuum models and Monte Carlo (MC) simulations to reproduce the experiments: $:^{5,7,18,22-24}$ stochastic roughening, $, 7,22-25$ geometrical shadowing, ${ }^{22}$ reemission of neutrals, ${ }^{23,24,26}$ micromasking by inhibitors, ${ }^{18,27}$ and ion reflection. . $^{5,25,28-30}$ Longitudinal striations or ripplelike structures (called the line edge/width roughness) often formed on feature sidewalls ${ }^{8-15}$ are usually appreciated to arise extrinsically from pattern transfer of the mask edge roughness under the effects of ion shadowing, ${ }^{12}$ in practice, they would also arise intrinsically or spontaneously from plasma-surface interactions themselves on feature sidewalls at high off-normal ion incidence, although little mechanistic work has been concerned with surface roughening and rippling in response to ion incidence angle during plasma etching. ${ }^{5,7,25,31-33}$

The morphological evolution of solid material surfaces at atomistic scale has also been of interest during ion beam sputtering (IBS); in particular, spontaneous or self-organized formation of ordered nanostructures such as periodic dots and ripples in response to ion incidence angle onto surfaces has been extensively studied since decades, ${ }^{34-39}$ as a promising approach to fabricate well-ordered nanostructures. 
The formation and evolution of different surface morphologies in IBS is often assumed to be due to a competition between surface roughening or instability (caused by curvaturedependent sputtering) ${ }^{40-43}$ and smoothing (caused by surface diffusion and/or viscous flow of surface atoms). ${ }^{44-47}$ Several mechanisms have been further invoked to reproduce the experiments, including shadowing, ${ }^{48}$ redeposition, ${ }^{49}$ mass redistribution, ${ }^{50-52}$ and ion reflection/channeling. ${ }^{53-56}$

Plasma etching and IBS are mechanistically similar in that surface erosion or material removal is affected critically by ion bombardment: the former results primarily from ion-enhanced etching through synergistic interactions of simultaneously incident ions (typically $50-500 \mathrm{eV}$ ) and neural reactants with surfaces, while the latter from physical sputtering only through ion bombardment (typically $\left.10^{3}-10^{5} \mathrm{eV}\right)$. However, mechanistic discussions of the surface morphology evolution are frequently disconnected between the two fields, although similar mechanisms have been invoked in both fields to reproduce the experiments as above; in particular, the role and effects of ion scattering or reflection from feature surfaces on incidence have not been fully understood in both fields, although they are recognized to be key to the formation and evolution of specific geometrical features therein.

In plasma etching, the ion reflection was taken into account in continuum models and MC simulations for feature profile evolution, usually assuming specular reflection from feature sidewalls, to reproduce microtrenches formed at the corner of the feature bottom; ${ }^{57-65}$ a few plasma beam and MC studies of Sawin et al. suggested the importance of the effects of ion reflection on surface roughening and rippling; $5,25,31-33$ the ion reflection was also suggested as a possible explanation for the evolution of surface roughness during pulsed plasma etching. $^{28}$ In IBS, Hauffe suggested that the coarsening of terrace-like (or sawtooth-like) ripple structures is caused by reflected ions that re-impinge on the adjacent facets, ${ }^{53}$ which was supported in several experiments ${ }^{66-69}$ while not in some other experimental ${ }^{70}$ and continuum model 71 studies; Bradley and Harper gave little more than comments on the effects of ion reflection on the ripple formation, in their pioneering theoretical study based on continuum equations; ${ }^{40}$ a few classical molecular dynamics (MD) simulations were concerned with the step-edge sputter yields at high off-normal ion incidence arising from the ion reflection and re-impingement and with their effects on the ion surface channeling and ripple formation thereat. ${ }^{54-56}$

We have investigated surface roughening and ripple formation during $\mathrm{Si}$ etching in $\mathrm{Cl}$-based plasmas:,72-78 our MC-based three-dimensional atomic-scale cellular model (ASCeM-3D), mechanistically including the effects of ion reflection, simulated the roughening and rippling in response to ion incidence angle $\theta_{i \cdot}{ }^{72,73}$ Moreover, the experiments of inductively coupled plasma (ICP) etching of $\mathrm{Si}$ in $\mathrm{Cl}_{2}$ with different ion incident energies of $E_{i} \approx 20-500 \mathrm{eV}\left(\theta_{i}=0^{\circ}\right)$ exhibited two modes of surface rougheing: ${ }^{74,75}$ roughening mode at low $E_{i}(<200-300 \mathrm{eV})$ and smoothing or non-roughening mode at higher $E_{i}$. A comparison of the experiments and MC simulations with the help of $\mathrm{MD}^{76,77}$ and plasma diagnostics ${ }^{74}$ suggested that the roughening-smoothing transition observed is characterized by reduced effects of the ion reflection, caused by a change in the predominant ion flux due to that in plasma conditions. ${ }^{74}$ Further experiments using sheath control plates to achieve the off-normal ion incidence onto substrate surfaces $\left(E_{i} \approx 500 \mathrm{eV} ; \theta_{i} \approx 40^{\circ}, 80^{\circ}\right.$ ) first demonstrated the formation of well-defined periodic surface ripples by plasma etching (though in the smoothing mode for normal $\theta_{i}=0^{\circ}$ ). ${ }^{78}$

In this paper, we elucidate quantitatively the crucial role of ion reflection in surface roughening and rippling during plasma etching, based on MC-based ASCeM-3D simulations of $\mathrm{Si}$ etching in Cl-based plasmas with $E_{i} \approx 20-500 \mathrm{eV}$ at $\theta_{i}=0^{\circ}-85^{\circ}$. The model includes the effects of ion reflection from feature surfaces on incidence (and/or its penetration into substrates) through calculating the momentum and energy conservation in successive two-body elastic collisions with substrate atoms every ion incidence (not assuming specular reflection). In this study, the "reflection coefficient $r_{i}\left(0 \leq r_{i} \leq 1\right)$ " is further introduced in the model, representing the fraction of ions incident on surfaces with the reflection/penetration calculation scheme turned on; preliminary results for normal $\theta_{i}=0^{\circ}$ were in part described previously. ${ }^{7,74,75}$ A quantitative understanding, under what conditions the reduced ion reflection $\left(r_{i}<1\right)$ results in surface smoothing/non-roughening and under what conditions it retains the roughening and rippling, would be of great technological as well as fundamental importance. Moreover, we discuss the ripple topography and dynamics (wavelength, amplitude, profile, and propagation velocity) at intermediate off-normal $\theta_{i}=45^{\circ}$ depending on the degree of ion reflection. These effects of reduced ion reflection on plasma-induced surface roughening and ripple formation are considered as effectively equivalent to those of enhanced smoothing due to neutral reactants, which competes with the roughening and rippling caused by ion bombardment.

\section{MODEL}

The ASCeM-3D is concerned with plasma-surface interactions and feature profile evolution during Si etching in Cl-based plasmas, which has been detailed previously. ${ }^{7,72,73}$ Briefly, the model takes into account the transport and surface reaction kinetics of $\mathrm{Cl}^{+}$ions, $\mathrm{Cl}$ and $\mathrm{O}$ neutrals, and etch/sputter products and byproducts of $\mathrm{SiCl}_{x}$ and $\mathrm{SiCl}_{x} \mathrm{O}_{y}$ in microstructural features. The simulation domain consists of small cubic cells of atomic size $\left(L=\rho_{s}{ }^{-1 / 3}=2.7 \AA\right.$ with $\rho_{s}=5.0 \times 10^{22} \mathrm{~cm}^{-3}$, the atomic density of $\mathrm{Si}$ substrates), where the evolving interfaces are represented by removing $\mathrm{Si}$ atoms from and/or allocating them to the substrate surface cells concerned. The transport of ions and neutrals is analyzed based on single-particle trajectories with periodic boundary conditions in horizontal directions, where the transport is calculated every movement of step $L$, taking into account the geometrical shadowing effects of the feature and those of surface reemission for neutrals. The particles are assumed to move straight without collisions with other particles in vacuum; then, the particles are assumed to reach the surface if there is a $\mathrm{Si}$ atom in any of the 26 cells neighboring the cell in which the particle concerned is. The local surface normal and thus the local angle $\theta$ of incidence (relative to it) 
on feature surfaces is calculated constantly using the extended four-point technique for $5 \times 5 \times 5$ neighboring cells (125 cells in total) at around the substrate surface cell that the particle reaches. Note that for primary incident ions, $\theta=\theta_{i}$ on flat or completely planar substrate surfaces, while generally $\theta \neq \theta_{i}$ on local surfaces of microstructural features.

Surface chemistry and kinetics include surface chlorination, chemical etching, ion-enhanced etching, sputtering, surface oxidation, redeposition of etch/sputter products desorbed from feature surfaces being etched, and deposition of etch/sputter byproducts coming from the plasma. The ionrelated etch/sputter yields depending on ion incident energy $E_{i}$ and angle $\theta$ are approximated by the functional form of the universal energy dependence: ${ }^{79} Y\left(E_{i}, \theta\right)=A\left(E_{i}{ }^{1 / 2}-E_{\mathrm{th}}^{1 / 2}\right)$ $g(\theta)$ at $E_{i} \geq E_{\mathrm{th}}$, where $Y$ is the total number of $\mathrm{Si}$ atoms desorbed from surfaces per ion impact, $A$ is a proportional constant or scaling factor (taken to rely on surface conditions ${ }^{7,72,73}$ ), and $E_{\mathrm{th}}$ is the threshold energy concerned. In plasma etching where the ion-enhanced etching reactions dominate over sputtering, the etch yield is assumed to be maximum at normal $\theta=0^{\circ}$, while the sputter yield peaks at off-normal $\theta \approx 60^{\circ}-70^{\circ}$. 7,72

The model also takes into account the ion reflection from feature surfaces on incidence into vacuum and/or the ion penetration into substrates. ${ }^{80,81}$ The ion reflection/penetration is analyzed every ion incidence, using 3D MC calculation of single-particle $\mathrm{Cl}^{+}$ion trajectories according to the momentum and energy conservation through successive two-body elastic collisions with the most neighboring substrate $\mathrm{Si}$ atoms; $;^{7,72,73}$ the ions reflected from the surface into vacuum (in some cases at high off-normal incidence, scattered out of the surface after channeling) are taken to move further toward another surface of the feature or go out of the simulation domain, similarly to neutrals reemitted. The interaction potential for $\mathrm{Cl}-\mathrm{Si}$ employed is the simple, repulsive Ziegler-Biersack-Littmark (ZBL) type. ${ }^{82,83}$

Simulations were made for square substrates $W=50 \mathrm{~nm}$ on a side having initially flat or completely planar surfaces (their root-mean-square roughness $\mathrm{RMS}=0$ at $t=0$ ), with different ion incident energies of $E_{i}=20-500 \mathrm{eV}$ and incidence angles of $\theta_{i}=0^{\circ}, 45^{\circ}$, and $75^{\circ}$ onto substrate surfaces (relative to the substrate surface normal). The following conditions were then assumed as in high-density plasma etching environments (unless otherwise stated): ${ }^{74,75}$ a directionally incoming ion flux $\Gamma_{i}^{0}=1.0 \times 10^{16} \mathrm{~cm}^{-2} \mathrm{~s}^{-1}$, ion temperature $k_{B} T_{i}=0.5 \mathrm{eV} \quad\left(R=E_{i} / k_{B} T_{i}\right.$ reflects the directionality or the angular distribution of incoming ion fluxes), isotropically incoming neutral reactant-to-ion flux ratio $\Gamma_{n}^{0} / \Gamma_{i}^{0}=100$, and neutral temperature $T_{g}=500 \mathrm{~K}$ in the absence of incoming etch inhibitors such as oxygen and byproducts $\left(\Gamma_{o}{ }^{0}=\Gamma_{p}{ }^{0}=0\right)$; in addition, the sticking probability $S_{q}=0.05$ was assumed for redeposition of etch/sputter products, along with the dopant concentration $N_{e}=1.0 \times 10^{18} \mathrm{~cm}^{-3}$ and surface temperature $T_{s}=320 \mathrm{~K}$ of substrates. Note that the effects of $N_{e}$ and $T_{s}$ are included in the model via the known reaction probability for $\mathrm{Cl}$-atom chemical etching of Si depending on $N_{e}$ and $T_{s}$ (obtained from experiments of Si etching in heated $\mathrm{Cl}_{2}$ gases). ${ }^{84}$ The etching or plasma exposure time was $1-2 \mathrm{~min}$ in this study [ion fluence $\Phi=\Gamma_{i}^{0} \times$ time $=(0.6$ $\left.-1.2) \times 10^{18} \mathrm{~cm}^{-2}\right]$, which is typical in industry.

\section{RESULTS AND DISCUSSION}

\section{A. Role of ion reflection in roughening and rippling}

Figure 1 shows typical surface features of $\mathrm{Si}$ at $t=60 \mathrm{~s}$ after the start of etching in $\mathrm{Cl}_{2}$ plasma (angle view, $50 \times 50 \mathrm{~nm}^{2}$ ), simulated with different ion reflection coefficients in the range $r_{i}=1-0$ for $E_{i}=100 \mathrm{eV}$ at $\theta_{i}=0^{\circ}, 45^{\circ}$, and $75^{\circ}$. The corresponding etch rate and rms surface roughness are shown in Fig. 2 as a function of $r_{i}$, together with the ion-enhanced etch rate $\mathrm{ER}_{\text {ion }}=Y_{\mathrm{SiCl}_{4}}\left(E_{i}, \theta\right) \Gamma_{i}^{0} / \rho_{s}$ of $\mathrm{Si}$ surfaces chlorine saturated bombarded by $\mathrm{Cl}^{+}$ions (assuming $\left.\theta=\theta_{i}\right){ }^{85,86}$ The "reflection coefficient $r_{i} \quad\left(0 \leq r_{i} \leq 1\right)$ " is defined as the fraction of ions incident on surfaces with the reflection/penetration calculation scheme turned on, or the coefficient $\left(1-r_{i}\right)$ is as the fraction of incident ions with the

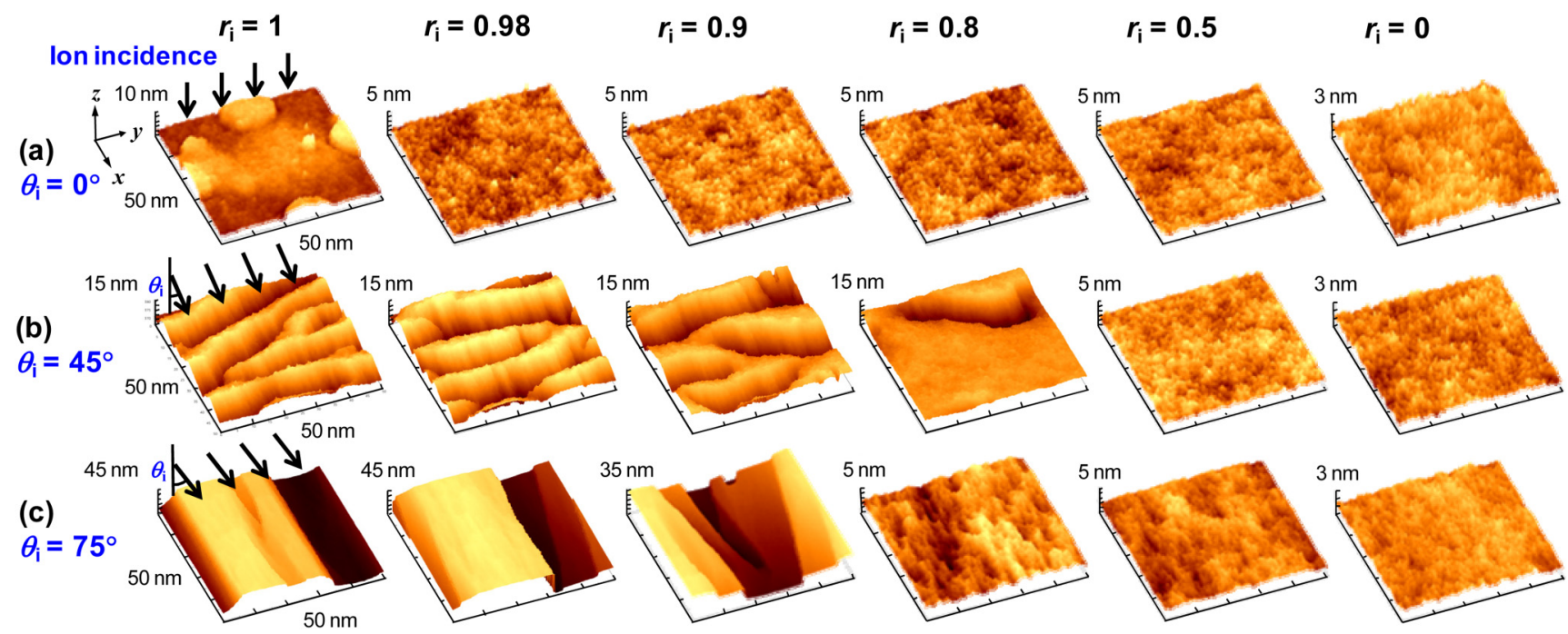

FIG. 1. Typical surface features of Si at $t=60 \mathrm{~s}$ after the start of etching in $\mathrm{Cl}_{2}$ plasma (angle view, $50 \times 50 \mathrm{~nm}^{2}$ ), simulated with different ion reflection coefficients in the range $r_{i}=1-0$ for an ion incident energy of $E_{i}=100 \mathrm{eV}$ at different incidence angles of $\theta_{i}=$ (a) $0^{\circ}$, (b) $45^{\circ}$, and (c) $75^{\circ}$ onto substrate surfaces. The coordinate system $(x, y, z)$ used in the model is inset in (a). 

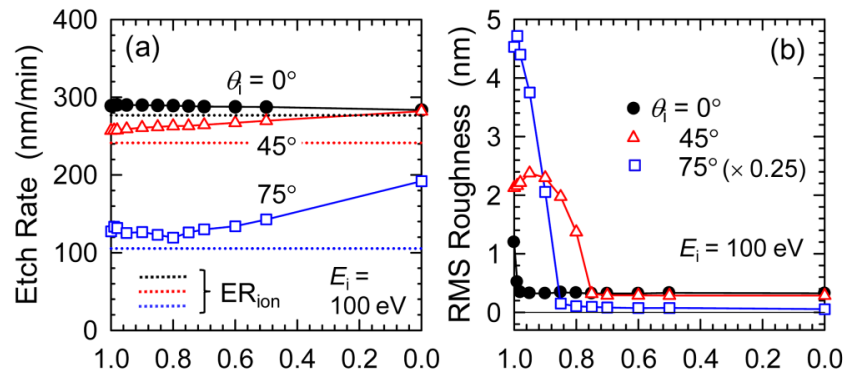

Ion Reflection Coefficient $r_{\mathrm{i}}$
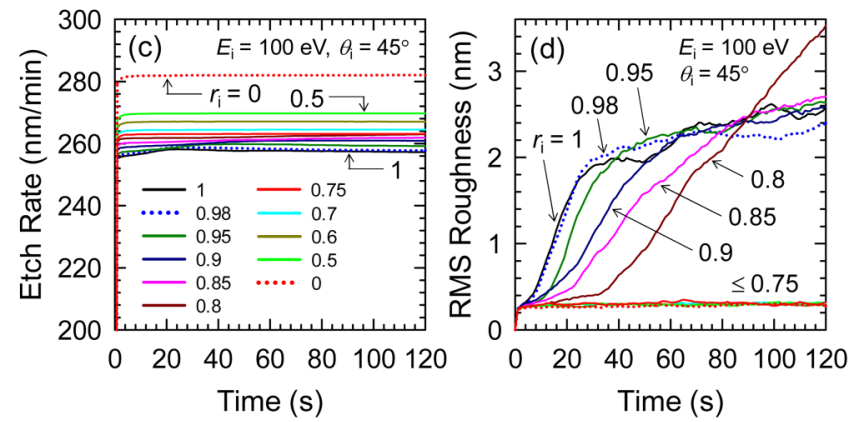

FIG. 2. (a) Etch rate and (b) rms surface roughness of $\mathrm{Si}$ at around $t=60 \mathrm{~s}$ as a function of ion reflection coefficient in the range $r_{i}=1-0$ for $E_{i}=100$ $\mathrm{eV}$ at different $\theta_{i}=0^{\circ}, 45^{\circ}$, and $75^{\circ}$, obtained from simulations as in Fig. 1. Also shown in (a) for reference is the ion-enhanced etch rate $\mathrm{ER}_{\text {ion }}=$ $Y_{\mathrm{SiCl}_{4}}\left(E_{i}, \theta\right) \Gamma_{i}^{0} / \rho_{s}$ of $\mathrm{Si}$ surfaces chlorine saturated (assuming $\theta=\theta_{i}$ ). (c) Etch rate and (d) rms roughness of $\mathrm{Si}$ as a function of time in the range $0 \leq$ $t \leq 120 \mathrm{~s}$ for different $r_{i}=1-0$ with $E_{i}=100 \mathrm{eV}$ at $\theta_{i}=45^{\circ}$, obtained from simulations as in Fig. 1(b). The data shown are the averages extracted from several runs of the simulation.

reflection/penetration calculation turned off; in other words, at least $\left(1-r_{i}\right) \times 100 \%$ of the incident ions are taken to stop and deposit their kinetic energy at the point of surfaces on incidence without reflection/penetration. Note that in ASCeM-3D, the ion reflection/penetration is calculated every ion incidence (as mentioned in Sec. II), giving internally the reflection probability $R_{i}\left(E_{i}, \theta\right)$ depending on ion incident energy and incidence angle along with the distributions of reflected energies and angles; ;,63 e.g., the reflectivity $R_{i}$ for $100-\mathrm{eV} \mathrm{Cl}^{+}$impacts onto bare $\mathrm{Si}$ surfaces is evaluated to increase from 0.01 at $\theta_{i}=0^{\circ}$ to $\geq 0.46$ at $\theta \geq 70^{\circ}$. Then, the probability of ion reflection in this study is assumed to be effectively given by $\mathfrak{R}_{\mathrm{i}}\left(E_{i}, \theta\right)=r_{i} \times R_{i}\left(E_{i}, \theta\right)$, where $0 \leq r_{i}$, $R_{i}, \mathfrak{R}_{\mathrm{i}} \leq 1$, and the coefficient $r_{i}$ may be assumed to be a measure of the reflectivity for ion species on $\mathrm{Si}$ surfaces (a second condition of interest) relative to that for $\mathrm{Cl}^{+}$on $\mathrm{Si}$ (base condition), as further discussed later with respect to Fig. 3. Note that the ions assumed under a second condition are virtual ones, in a sense, having the same mass, interaction potential, and thus collision dynamics (such as incident energy and angle dependences of the reflection probability, and energy and angle distributions of reflected atoms/molecules) as $\mathrm{Cl}^{+}$except for the magnitude of the reflectivity.

Simulations with conventional $r_{i}=1^{7,72,73}$ exhibit the random roughness at normal incidence $\left(\theta_{i}=0^{\circ}\right)$; in contrast, they exhibit sawtooth-like ripples with their wave vector oriented parallel (crests/troughs elongated perpendicular) to the direction of ion incidence at intermediate off-normal angles $\left(15^{\circ}<\theta_{i}<60^{\circ}\right)$, while striations or ripplelike structures with the wave vector perpendicular (crests/troughs parallel) to it at high off-normal angles $\left(70^{\circ}<\theta_{i}<85^{\circ}\right)$.

As the reflection coefficient $r_{i}$ is slightly decreased from unity, the roughness is reduced and the ripple structures tend to fade away: the rms roughness decreases substantially down to the low level of stochastic roughening (RMS $<0.3 \mathrm{~nm}$, slightly larger at higher $E_{i}$ and lower $\theta_{i}$ ) for decreased $r_{i} \leq r_{i}^{*} \approx 0.95,0.75$, and 0.8 at $\theta_{i}=0^{\circ}, 45^{\circ}$, and $75^{\circ}$, respectively, showing no pronounced ripple structures at off-normal $\theta_{i}$. Note that the low-level stochastic roughening is due to the uniformity of incident fluxes of ions and/or neutral etchants on surfaces at microscale, and thus its degree depends slightly on plasma conditions such as $E_{i}, \theta_{i}, \Gamma_{n}^{0} / \Gamma_{i}^{0}$, and $T_{s}{ }^{7}$ The rms roughness does not scale with the etch rate, which tends to be increased slightly with decreasing $r_{i}$ owing to the increased ion energy deposited on surfaces on incidence; the etch rate is larger at $\theta_{i}=0^{\circ}$ and $45^{\circ}$ than at $\theta_{i}=$ $75^{\circ}$, reflecting the angle dependence of the etch yield $Y_{\mathrm{SiCl}_{4}}\left(E_{i}, \theta\right)$ taken in the model. In simulations, as also shown in Fig. 2 partly for $\theta_{i}=45^{\circ}$, the rms roughness for $r_{i}>r_{i}^{*}$ exhibits a gradual increase with time to reach quasi-steady state in $t<120 \mathrm{~s}$ at $\theta_{i}=0^{\circ}$ and $45^{\circ}$, while it exhibits a continuous increase during etching at $\theta_{i}=75^{\circ} ;, 7,72,73$
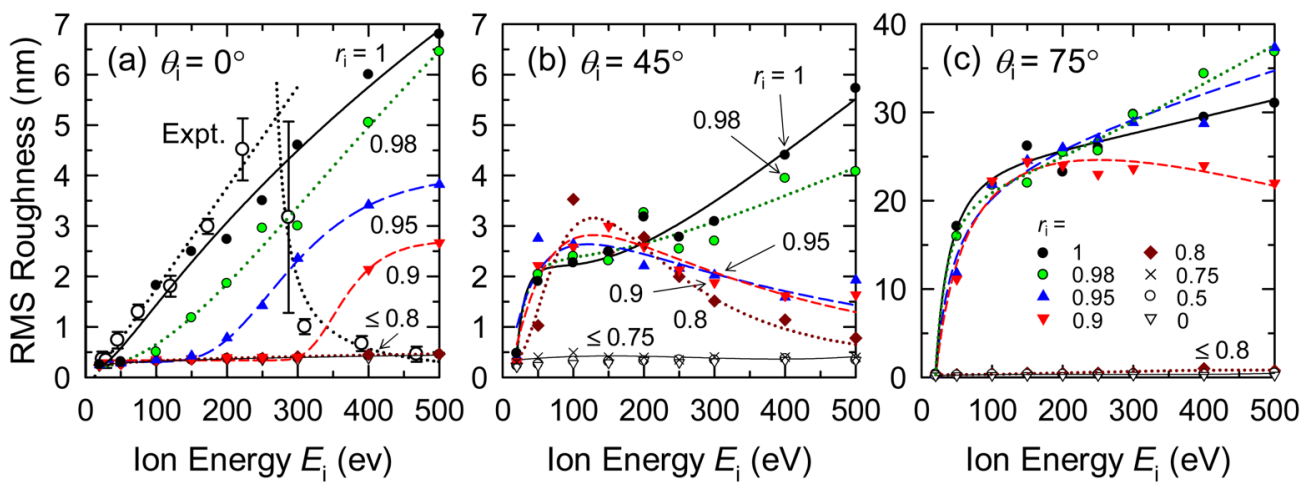

FIG. 3. RMS surface roughness of $\mathrm{Si}$ at around $t=120 \mathrm{~s}$ as a function of ion incident energy in the range $E_{i}=20-500 \mathrm{eV}$, obtained from simulations of $\mathrm{Si}$ etching in $\mathrm{Cl}_{2}$ plasma for different reflection coefficients of $r_{i}=1-0$ at different incidence angles of $\theta_{i}=$ (a) $0^{\circ}$, (b) $45^{\circ}$, and (c) $75^{\circ}$. Note that the low-energy conditions of $E_{i}=20 \mathrm{eV}$ at any $\theta_{i}$ and $E_{i}=50 \mathrm{eV}$ at $\theta_{i}=0^{\circ}$ do little roughening on their own even for $r_{i}=1$. The data shown are the averages extracted from several runs of the simulation, and the solid, dashed, and dotted lines in the respective figures are for guiding the eyes only. Additionally shown in (a) for comparison is the rms roughness experimentally obtained in $\mathrm{ICP} \mathrm{Cl}_{2}$ plasma etching of $\mathrm{Si}^{74}$ 
in contrast, for $r_{i} \leq r_{i}{ }^{*}$ at any $\theta_{i}$, the roughness retains the low level during etching after only a little increase at the initial stage; in addition, the etch rate reaches quasi-steady state in $t<$ a few seconds soon after the start of etching, irrespective of $r_{i}$ and $\theta_{i}$.

These results clearly indicate the crucial role of ion scattering or reflection from feature surfaces on incidence in surface roughening and ripple formation during plasma etching: it is indispensable therein, and the degree of roughening and rippling relies significantly on the reflectivity of ions. In effect, irrespective of macroscopic incidence angle $\theta_{i}$ onto substrate surfaces, the ions are expected to be often incident on sidewalls of roughness features at locally high offnormal angles $\theta$; such off-normally incident ions are then reflected therefrom and tend to re-impinge on the neighboring sidewalls and/or bottom surfaces of the feature, give rise to significant momentum and energy transfer thereto, cause enhanced etching of roughness features, and thus enhance their degree of roughness to its lateral as well as vertical extent and also induce the ripple formation.

Figure 3 shows the rms surface roughness of $\mathrm{Si}$ at around $t=120 \mathrm{~s}$ as a function of ion energy in the range $E_{i}=20-500 \mathrm{eV}$, obtained from simulations of $\mathrm{Si}$ etching in $\mathrm{Cl}_{2}$ plasma for different reflection coefficients of $r_{i}=1-0$ at $\theta_{i}=0^{\circ}, 45^{\circ}$, and $75^{\circ}$. The rms roughness for conventional $r_{i}=1$ is increased with increasing $E_{i}$ irrespective of $\theta_{i}$, where low-energy conditions of $E_{i}=20 \mathrm{eV}$ at any $\theta_{i}$ and $E_{i}=50 \mathrm{eV}$ at $\theta_{i}=0^{\circ}$ do little roughening on their own even for $r_{i}=1$; note that this is not inconsistent with the experiments of atomic layer etching of $\mathrm{Si}^{1,2}$ where low-energy $\mathrm{Ar}^{+}$ions impact on chlorinated surfaces $\left(E_{i} \approx 50 \mathrm{eV}, \theta_{i}=0^{\circ}\right)$. The increase in roughness with increasing $E_{i}$ has been observed in some experiments of plasma etching of $\mathrm{Si}$ in $\mathrm{Cl}_{2},{ }^{87}$ while some other experiments showed a decrease in roughness with increasing $E_{i}$ during plasma etching of $\mathrm{Si}$ in $\mathrm{Cl}_{2}{ }^{88}$ and $\mathrm{SF}_{6} .{ }^{89,90}$

The decrease in roughness (or the smoothing/nonroughening) with reduced ion reflection tends to be less significant at increased $E_{i}$ and $\theta_{i}$ : the higher the $E_{i}$ and the $\theta_{i}$, the smaller is the critical $r_{i}^{*}$ giving the rms roughness decreased down to the low level of stochastic roughening (RMS $<0.3-0.5 \mathrm{~nm}$ ); concretely, $r_{i}^{*} \approx 0.95-0.8$ (decreasing significantly with increasing $E_{i}$ ) at normal $\theta_{i}=0^{\circ}$, while $r_{i}^{*} \approx 0.8-0.75$ (almost independent of $E_{i}$ investigated) at offnormal $\theta_{i}=45^{\circ}$ and $75^{\circ}$. In practice, the effects of ion reflection and thus the effects of reduced ion reflection are expected to be less significant for 2D ripples at $\theta_{i}=45^{\circ}$ and $75^{\circ}$ than for $3 \mathrm{D}$ random roughness features at $\theta_{i}=0^{\circ}$, because the flux of ions reflected from feature sidewalls and then re-impinging on the neighboring deeper surfaces of the feature ${ }^{59,62}$ is assumed to be smaller for 2D features (such as trenches, opened in one direction) than for 3D features (such as holes/vias, surrounded by walls on all sides). The reduced effects of ion reflection followed by re-impingement are also expected to be effectively less significant at increased $E_{i}$, where the increased directionality of incoming ion fluxes is assumed to increase the flux of ions directly incident on the bottom of roughness features, and thus to decrease the flux of ions reflected from feature sidewalls. In addition, the reduced effects of ion reflection at $\theta_{i}=75^{\circ}$ would be slightly significant as compared to those at $\theta_{i}=45^{\circ}\left(r^{*} \approx 0.75\right.$ and 0.8 at $\theta_{i}=45^{\circ}$ and $75^{\circ}$, respectively), presumably owing to the contribution of the ion scattering-caused channeling effects at high off-normal incidence.

The critical $r_{i}^{*}$ values at normal $\theta_{i}=0^{\circ}$ indicate that for decreased $r_{i} \leq 0.8$, the rms roughness retains the low level of stochastic roughening even at high $E_{i}>400 \mathrm{eV}$ for $\theta_{i}=0^{\circ}$. This is consistent with our ICP plasma etching experiments of $\mathrm{Si}$ in $\mathrm{Cl}_{2}$ exhibiting the roughening-smoothing transition at increased $E_{i}>200-300 \mathrm{eV},{ }^{7,74,75}$ as additionally shown in Fig. 3(a): the rms roughness in experiments increases with increasing $E_{i}$ at low $E_{i}<200-300 \mathrm{eV}$ (roughening mode, scaling with the etch rate), which is well reproduced by the simulations with conventional $r_{i}=1$; on the other hand, at further increased $E_{i}$, it decreases substantially with $E_{i}$ down to the low level (smoothing mode, not scaling with the etch rate), which is not reproduced by them, but reproduced by simulations with decreased $r_{i} \leq 0.8$. Note that in our experiments, the etch rate was observed to increase with increasing $E_{i}$ over the $E_{i}$ range investigated (both roughening and smoothing modes), which is reproduced easily by the simulations with any $r_{i}=1-0$ [the numerical etch rate $\mathrm{ER} \approx$ $\mathrm{ER}_{\text {ion }} \propto E_{i}^{1 / 2}$ is relatively insensitive to $r_{i}$ as partly seen in Figs. 2(a) and 2(c)]. ${ }^{7,74}$

Correspondingly, the predominant ion flux was found to change from reactive $\mathrm{Cl}_{2}{ }^{+} / \mathrm{SiCl}_{3}{ }^{+}$at low $E_{i}$ (base condition) to depositive $\mathrm{SiCl}^{+}$at increased $E_{i}$ (a second condition). In effect, as the ion energy $E_{i}$ was increased in experiments, ${ }^{7,74}$ the fluxes of ionized etch products/byproducts $\mathrm{SiCl}_{x}{ }^{+}(x=0-4)$ were observed to increase owing to the increase in etch rate, while those of feed gas ions $\mathrm{Cl}_{x}^{+}(x=1,2)$ were to decrease partly owing to the reduced partial pressures or concentrations of feed gases under operating conditions of constant pressure; as a result, the predominant ion flux during etching was $\mathrm{Cl}_{2}^{+}$(followed by $\mathrm{SiCl}_{3}{ }^{+}, \mathrm{SiCl}^{+}$, and $\mathrm{SiCl}_{4}{ }^{+}$) at low $E_{i}<$ $30 \mathrm{eV}, \mathrm{SiCl}_{3}{ }^{+}$(followed by $\mathrm{Cl}_{2}{ }^{+}, \mathrm{SiCl}^{+}$, and $\mathrm{SiCl}_{4}{ }^{+}$) at $30<$ $E_{i}<50 \mathrm{eV}, \mathrm{SiCl}_{3}{ }^{+}$(followed by $\mathrm{SiCl}^{+}, \mathrm{Cl}_{2}{ }^{+}$, and $\mathrm{SiCl}_{4}{ }^{+}$) at $50<E_{i}<120 \mathrm{eV}$, and then $\mathrm{SiCl}^{+}$(followed by $\mathrm{SiCl}_{3}{ }^{+}, \mathrm{SiCl}_{4}{ }^{+}$, and $\mathrm{Cl}_{2}{ }^{+}$) at further increased $E_{i}>120 \mathrm{eV}$. At high $E_{i}>300$ $\mathrm{eV}$, the $\mathrm{SiCl}^{+}, \mathrm{SiCl}_{3}{ }^{+}, \mathrm{SiCl}_{4}{ }^{+}$, and $\mathrm{Cl}_{2}{ }^{+}$fluxes were relatively $1,<0.3,<0.06$, and $<0.02$, respectively. Such an aspect of the predominant ionic species $\left(\mathrm{SiCl}_{x}^{+}\right.$and not $\mathrm{Cl}_{x}^{+}$) during plasma etching of $\mathrm{Si}$ in $\mathrm{Cl}$-based plasmas has been predicted by recent modeling simulations, ${ }^{91}$ being attributable partly to the total electron-impact ionization cross section for $\mathrm{SiCl}_{x}$ $(x=1-4)$ molecules, which is about 2-3 times larger than that for $\mathrm{Cl}_{2}$.

According to MD simulations of $\mathrm{Cl}_{x}^{+}(x=1,2)$ and $\mathrm{SiCl}_{x}^{+}(x=0-4)$ ion impacts on $\mathrm{Si}$ surfaces with $E_{i}=20$ $-500 \mathrm{eV}$ at $\theta=0^{\circ}-85^{\circ}, 7,77$ incident molecular ions often fragment or dissociate upon impact. $\mathrm{In}^{+}, \mathrm{Cl}_{2}{ }^{+}, \mathrm{SiCl}^{+}$, and $\mathrm{SiCl}_{3}{ }^{+}$incidences, e.g., the atoms/molecules scattered or reflected directly from the surface on incidence during impact are $\mathrm{Cl}$ for $\mathrm{Cl}^{+}$impact, $\mathrm{Cl}$ and $\mathrm{Cl}_{2}$ for $\mathrm{Cl}_{2}{ }^{+}$impact, $\mathrm{Cl}, \mathrm{Si}$, and $\mathrm{SiCl}$ for $\mathrm{SiCl}^{+}$impact, and $\mathrm{Cl}, \mathrm{Cl}_{2}, \mathrm{Si}, \mathrm{SiCl}, \mathrm{SiCl}_{2}$, and $\mathrm{SiCl}_{3}$ for $\mathrm{SiCl}_{3}{ }^{+}$impact, including fragments upon impact. The fraction of incident molecular ions that are dissociated upon impact increases with increasing ion incident energy $E_{i}$, and for a given $E_{i}$, it peaks at around $\theta \approx 70^{\circ}$. In these situations, 
the reflection probability $R_{i}\left(E_{i}, \theta\right)$ for atomic $\mathrm{Cl}^{+}$impact is generally calculated as the total number of reflected $\mathrm{Cl}$ atoms divided by that of incident ones, while the probability for molecular $\mathrm{Cl}_{2}{ }^{+}, \mathrm{SiCl}^{+}$, and $\mathrm{SiCl}_{3}{ }^{+}$impacts is calculated in our MD simulations taking into account the effects of dissociation upon impact, as will be detailed elsewhere. ${ }^{77}$

The reflection probability $R_{i}\left(E_{i}, \theta\right)$ thus calculated tends to be lower for depositive $\mathrm{SiCl}^{+}$than for reactive $\mathrm{Cl}^{+}, \mathrm{Cl}_{2}{ }^{+}$, and $\mathrm{SiCl}_{3}{ }^{+} ;$,77 e.g., the reflectivity $R_{i}$ for $E_{i}=300 \mathrm{eV}$ ion impacts increases from 0.061 at $\theta=0^{\circ}$ to $\geq 0.54$ at $\theta \geq 70^{\circ}$ for $\mathrm{Cl}^{+}$, from 0.11 at $\theta=0^{\circ}$ to $\geq 0.73$ at $\theta \geq 70^{\circ}$ for $\mathrm{Cl}_{2}{ }^{+}$, from 0.14 at $\theta=0^{\circ}$ to $\geq 0.83$ at $\theta \geq 70^{\circ}$ for $\mathrm{SiCl}_{3}{ }^{+}$, and from 0.063 at $\theta=0^{\circ}$ to $\geq 0.67$ at $\theta \geq 70^{\circ}$ for $\mathrm{SiCl}^{+}$; note that $R_{i}$ is lower for higher $E_{i}$ owing to increased penetration at normal and off-normal incidence angles of approximately $\theta<70^{\circ}$, while it tends to be higher for higher $E_{i}$ at high off-normal $\theta>70^{\circ}$. Thus, the ratio of the reflectivity $R_{i}$ for $\mathrm{SiCl}^{+}$to that for $\mathrm{Cl}_{2}{ }^{+} / \mathrm{SiCl}_{3}{ }^{+}$is on the order of $\chi_{i} \approx 0.8-0.9$ at $\theta>70^{\circ}$, e.g., for $E_{i}=300 \mathrm{eV}\left(\chi_{i}\right.$ tends to be larger for higher $E_{i}$ ), which reflects the smoothing mode in experiments $\left(\theta_{i}=0^{\circ}\right)$ reproduced by ASCeM-3D with decreased $r_{i}$ as in Fig. 3(a). In addition, the smaller $r_{i}^{*} \approx 0.8-0.75$ at off-normal $\theta_{i}=45^{\circ}$ and $75^{\circ}$ (as compared to $r_{i}^{*} \approx 0.95-0.8$ at normal $\theta_{i}=0^{\circ}$ ) is also consistent with our recent experiments of $\mathrm{Si}$ etching in ICP $\mathrm{Cl}_{2}$ plasmas using sheath control plates: ${ }^{.78}$ the ripple formation was demonstrated under conditions of $E_{i} \approx 500 \mathrm{eV}$ with $\theta_{i} \approx$ $40^{\circ}$ and $80^{\circ}$, where the predominant ions were $\mathrm{SiCl}^{+}$with relatively low $R_{i}$, giving the smoothing mode for $\theta_{i}=0^{\circ}$.

\section{B. Similarity between reduced ion reflection and enhanced smoothing}

Similar smoothing/non-roughening behavior was observed in ASCeM-3D simulations with $r_{i}=1$, through increasing the neutral reactant flux $\left(\Gamma_{n}{ }^{0} / \Gamma_{i}{ }^{0}=50-1000\right)^{73}$ and the surface temperature $\left(T_{s}=320-500 \mathrm{~K}\right),{ }^{92}$ although it is mechanistically different from the smoothing with reduced ion reflection discussed above in Sec. III A: the roughness is reduced and the ripples tend to fade away as $\Gamma_{n}{ }^{0} / \Gamma_{i}^{0}$ and $T_{s}$ are increased, which is also less significant at increased $E_{i}$ and $\theta_{i}$. As $\Gamma_{n}{ }^{0} / \Gamma_{i}^{0}$ is increased for a given ion energy $E_{i}=100 \mathrm{eV}$ (with $T_{s}=320 \mathrm{~K}$ ), ${ }^{73}$ e.g., the rms roughness increases (scaling with the etch rate) at low $\Gamma_{n}^{0} / \Gamma_{i}^{0}<50$ irrespective of the incidence angle $\theta_{i}$. Then, the roughness at $\theta_{i}=0^{\circ}$ peaks at $\Gamma_{n}{ }^{0} / \Gamma_{i}^{0} \approx 50$ and then decreases substantially down to the low level of stochastic roughening at $\Gamma_{n}{ }^{0} / \Gamma_{i}^{0}>200$; the roughness at $\theta_{i}=45^{\circ}$ remains almost constant at $\Gamma_{n}{ }^{0} / \Gamma_{i}{ }^{0}>50$ and tends to decrease at $\Gamma_{n}{ }^{0} / \Gamma_{i}{ }^{0}>500$; the roughness at $\theta_{i}=75^{\circ}$ continues to increase and remains almost constant at $\Gamma_{n}^{0} / \Gamma_{i}^{0}>500$. The ripples at $\theta_{i}=45^{\circ}$ are significant in the range $\Gamma_{n}^{0} / \Gamma_{i}^{0} \approx 50-500$, and they tend to fade away at further increased $\Gamma_{n}{ }^{0} / \Gamma_{i}^{0}$, where the ripple wavelength is shortest at $\Gamma_{n}^{0} / \Gamma_{i}^{0} \approx 100$; the ripples at $\theta_{i}=75^{\circ}$ enlarge significantly at increased $\Gamma_{n}^{0} / \Gamma_{i}^{0}>100$, exhibiting a wide, deep groove. Moreover, as $T_{s}$ is increased for a given $E_{i}=100 \mathrm{eV}$ (with $\Gamma_{n}^{0} / \Gamma_{i}^{0}=100$ ), ${ }^{92}$ e.g., the rms roughness decreases substantially down to the low level at $T_{s}>360 \mathrm{~K}$ for $\theta_{i}=0^{\circ}$ and at $T_{s}>500 \mathrm{~K}$ for $\theta_{i}=45^{\circ}$ and $75^{\circ}$. Correspondingly, the ripples at $\theta_{i}=45^{\circ}$ and $75^{\circ}$ tend to fade away at increased $T_{s}$, where the wavelength of the former is increased with increasing $T_{s}$. In practice, the surface smoothing with increased $\Gamma_{n}^{0} / \Gamma_{i}^{0}\left(\theta_{i}=0^{\circ}\right)$ has been observed in some experiments of plasma etching of $\mathrm{Si}$ in $\mathrm{SF}_{6},{ }^{89}$ which showed a decrease in roughness with increasing pressure (thus probably increasing the flux of incoming neutral reactants); on the other hand, some other experiments showed an increase in roughness with increasing pressure during plasma etching of $\mathrm{Si}$ in $\mathrm{Cl}_{2}{ }^{88}$ and $\mathrm{SF}_{6} \cdot{ }^{90}$ The smoothing with increased $T_{s}$ has not so far been observed in experiments, which showed no $T_{s}$ dependence of the roughness during plasma etching of $\mathrm{Si}$ in $\mathrm{SF}_{6}$ at $T_{s}=-100$ to $40{ }^{\circ} \mathrm{C}^{89}$ and in $\mathrm{Cl}_{2}$ at $T_{s}=20-200{ }^{\circ} \mathrm{C} .{ }^{74}$

The decrease in roughness with increasing $\Gamma_{n}^{0} / \Gamma_{i}^{0}$ and $T_{s}$ (not scaling with the etch rate) is attributed to increased chemical effects of neutral reactants (chemical and ionenhanced etching reactions) on top of roughness features: ${ }^{7}$ the incident flux of neutral reactants is assumed to be smaller at the bottom than at the top of roughness features under shadowing effects of the feature, so the top of the features tends to etch faster than their bottom under conditions that the feature bottom starves for neutrals, which suppresses the evolution of surface roughness and ripples or causes surface smoothing. In effect, the smoothing with increased $\Gamma_{n}^{0} / \Gamma_{i}^{0}$ and $T_{s}$ is less significant for $2 \mathrm{D}$ ripples at $\theta_{i}=45^{\circ}$ and $75^{\circ}$ than for $3 \mathrm{D}$ random roughness features at $\theta_{i}=0^{\circ}$, because the shadowing effects for neutrals are lower for $2 \mathrm{D}$ than for $3 \mathrm{D}$ features. $^{93-95}$ The smoothing with increased $\Gamma_{n}{ }^{0} / \Gamma_{i}{ }^{0}$ and $T_{s}$ is also less significant at increased $E_{i}$, where the increased directionality of incoming ion fluxes is assumed to increase the flux of ions directly incident on the bottom of roughness features that starves for neutrals, and thus to effectively lower the effects of neutral shadowing.

These similarities would be no wonder between the effects of reduced ion reflection and those of increased neutral reactant flux and surface temperature, because the formation and evolution of surface roughness (and ripples) during plasma etching as well as IBS is assumed to be due to a competition between roughening (and rippling) and smoothing: ${ }^{7}$ the former is caused primarily by ion-enhanced etching reactions through ion bombardment, whose rates tend to be higher at the bottom of roughness features than at their top in the presence of ion reflection followed by re-impingement, unless the feature bottom starves for neutrals under shadowing; and the latter is by chemical effects of neutral reactants, which are more significant on top of roughness features under shadowing. In other words, the surface roughening (and rippling) occurs when the roughening dominates over smoothing through enhanced roughening and/or reduced smoothing, and the surface smoothing/non-roughening occurs when the smoothing dominates over roughening through reduced roughening and/or enhanced smoothing. Thus, in terms of smoothing, the reduced ion reflection (leading to reduced roughening) should be effectively equivalent to the increased neutral reactant flux and surface temperature (leading to enhanced smoothing).

This understanding gives an insight into the reason why the plasma-induced formation and evolution of roughness and ripples is so highly sensitive to the degree of ion reflection as in Figs. 1-3: small deviations of the reflection coefficient $r_{i}$ 
from unity result in smoothing/non-roughening (with no ripples at off-normal $\theta_{i}$ ), which is due to the chemical or smoothing effects of neutral reactants that are incident preferentially on top of roughness features under the effects of shadowing. Then, the deviation of the critical $r_{i}^{*}$ from unity should be much smaller (or the critical $r_{i}^{*}$ is larger, closer to unity) with increasing the effects of smoothing and/or decreasing those of roughening; on the contrary, the deviation should be larger (or the critical $r_{i}^{*}$ is much smaller than unity) with decreasing smoothing effects and/or increasing those of roughening (as in Fig. 3 for increased $E_{i}$ ). In effect, additional simulations made by varying $r_{i}$ with different $\Gamma_{n}^{0} / \Gamma_{i}^{0}$ and $T_{s}$ (for a given $E_{i}$ and $\theta_{i}$ with $\left.\Gamma_{o}^{0} / \Gamma_{i}^{0}=0\right)$, not presented here, showed that the decrease in roughness (or the smoothing/nonroughening) with reduced ion reflection tends to be less significant at decreased $\Gamma_{n}^{0} / \Gamma_{i}^{0}$ and $T_{s}$ : the lower the $\Gamma_{n}{ }^{0} / \Gamma_{i}^{0}$ and the $T_{\mathrm{s}}$, the smaller is the critical $r_{i}^{*}$ giving the rms roughness decreased down to the low level of stochastic roughening. In addition, simulations made by varying $r_{i}$ with different inhibitor oxygen-to-ion flux ratios $\left(\Gamma_{o}^{0} / \Gamma_{i}^{0}=0-0.5,{ }^{73}\right.$ for a given $E_{i}$ and $\theta_{i}$ with $\Gamma_{n}^{0} / \Gamma_{i}^{0}=100$ and $T_{s}=320 \mathrm{~K}$ ) further showed that the decrease in roughness with reduced ion reflection tends to be less significant at increased $\Gamma_{o}^{0} / \Gamma_{i}^{0}$ : the higher the $\Gamma_{o}{ }_{o}^{0} / \Gamma_{i}^{0}$, the smaller is the critical $r_{i}^{*}$, as expected from the effects of a small amount of incoming inhibitor flux that enhances the evolution of surface roughness or causes surface roughening (leading to the formation of columnar nanostructures like pillars at $\theta_{i}=0^{\circ}$ ) through micromasking via local passivation layer formation on top of roughness features of $\mathrm{Si}$ under shadowing. $5,7,18,27,73$

\section{Effects of reduced ion reflection on ripple topography and dynamics}

The effects of reduced ion reflection on ripple topography and dynamics would also be intriguing, since the surface features at intermediate off-normal $\theta_{i}=45^{\circ}$ in Fig. 1(b) appear to exhibit the ripple wavelength increased as the ion reflection coefficient $r_{i}$ is decreased (or equivalently, the smoothing is enhanced). Similar tendencies were also observed in ASCeM-3D simulations with $r_{i}=1$, not presented here, through increasing the neutral reactant flux ${ }^{73}$ and surface temperature. ${ }^{92}$ Moreover, in IBS studies, the enhanced smoothing has been known to lead to an increase in the wavelength of parallel-mode ripples (wave vector parallel, or wave crests/troughs perpendicular, to the direction of ion incidence) at lower beam fluxes and higher substrate temperatures: ${ }^{36,96,97} 0.8-\mathrm{keV} \mathrm{Ar}^{+}$beams onto $\mathrm{Cu}$ at $\theta_{i}=70^{\circ}$ with $\Gamma_{i}^{0}=(2.1-6.7) \times 10^{14} \mathrm{~cm}^{-2} \mathrm{~s}^{-1}$ and $T_{s}=393$ $-472 \mathrm{~K}$ showed the ripple wavelength of $\lambda_{r} \approx 300-2000 \mathrm{~nm}$ increasing with decreasing $\Gamma_{i}{ }^{0}$ and increasing $T_{s},{ }^{96}$ these experimental observations were reproduced by continuum models and MC simulations, suggesting that a competition between ion-induced roughening via sputtering and thermal smoothing via surface diffusion of mobile defects is responsible for the enhanced smoothing. ${ }^{36,96,97}$

Figure 4 shows the time evolution of surface features of $\mathrm{Si}$ (top view, $50 \times 50 \mathrm{~nm}^{2}$ ) during etching in $\mathrm{Cl}_{2}$ plasma
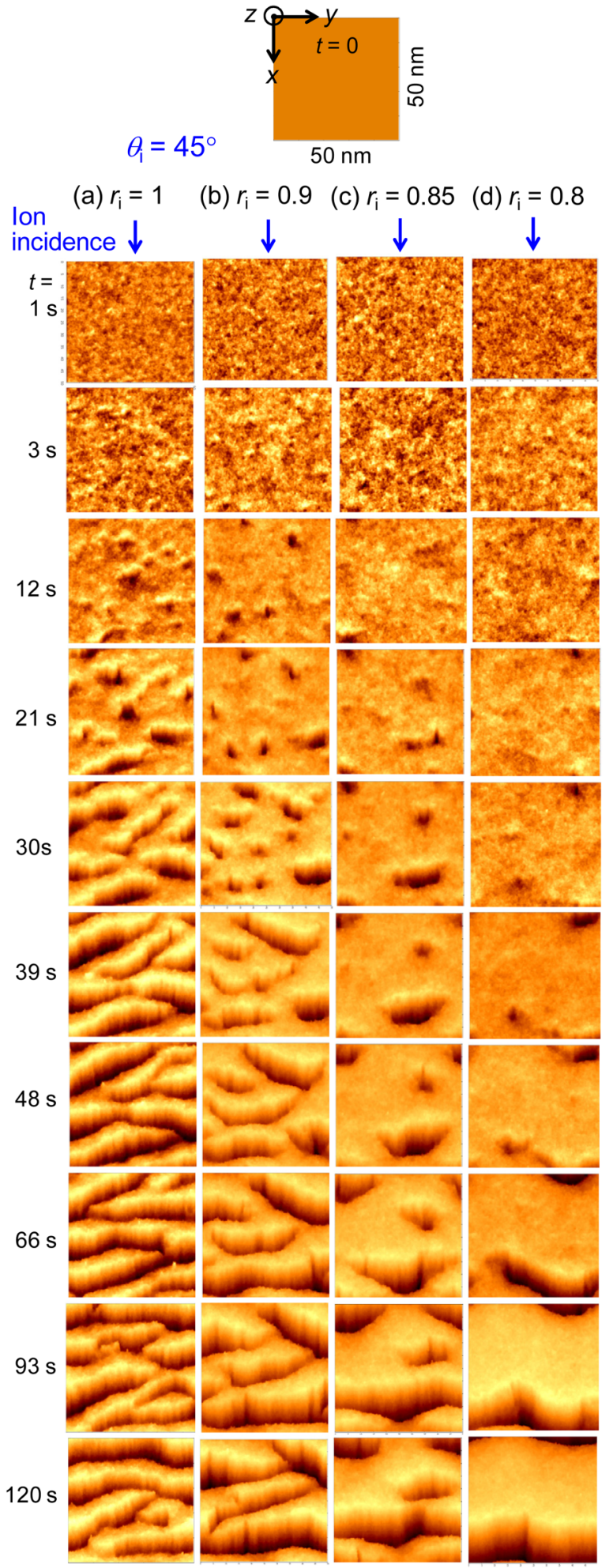

FIG. 4. Time evolution of surface features of Si (top view, $50 \times 50 \mathrm{~nm}^{2}$ ) during etching in $\mathrm{Cl}_{2}$ plasma $(0 \leq t \leq 120 \mathrm{~s})$ for different ion reflection coefficients of $r_{i}=$ (a) 1 , (b) 0.9 , (c) 0.85 , and (d) 0.8 with $E_{i}=100 \mathrm{eV}$ at $\theta_{i}$ $=45^{\circ}$, extracted from simulations as in Fig. 1(b). The coordinate system $(x$, $y, z$ ) used in the model is also inset here (as in Fig. 1), and the time evolution of the etch rate and rms surface roughness concerned here is the same as in Figs. 2(c) and 2(d). Note that the surface features shown are their snapshots taken every $T=W / v_{r} \approx 9 \mathrm{~s}$ and/or its multiples (18 and $\left.27 \mathrm{~s}\right)$ in the range $3 \leq t \leq 120 \mathrm{~s}$, where $W=50 \mathrm{~nm}$ is the side length of the square substrates being simulated, and $v_{r} \approx 5.5 \mathrm{~nm} / \mathrm{s}$ is the traveling velocity of surface features in the direction of ion incidence (or in the $x$-direction) during etching. 
$(0 \leq t \leq 120 \mathrm{~s})$ for different $r_{i}=1,0.9,0.85$, and 0.8 with $E_{i}=100 \mathrm{eV}$ at $\theta_{i}=45^{\circ}$, extracted from simulations as in Fig. 1(b). The corresponding representative side or crosssectional views of surface features are shown in Fig. 5, where the line of sight is perpendicular to the direction of ion incidence (or in the $y$-direction), and the simulation domain shown is $2 \mathrm{~nm}$ in width (in the $y$-direction at around the $x$ axis at $y=22,12,40$, and $40 \mathrm{~nm}$ for $r_{i}=1,0.9,0.85$, and 0.8 , respectively). The coordinate system $(x, y, z)$ used in ASCeM-3D is inset in Fig. 4 (also in Fig. 1), and the time evolution of the etch rate and rms surface roughness concerned is the same as earlier in Figs. 2(c) and 2(d). Note that in Figs. 4 and 5, the top- and side-view images of surface features shown are their snapshots taken every $9 \mathrm{~s}$ and/or its multiples (18 and $27 \mathrm{~s}$ ) in the range $3 \leq t \leq 120 \mathrm{~s}$. In the present situations, the surface features or ripple patterns move or travel laterally with the velocity of approximately $v_{r} \approx 5.5 \mathrm{~nm} / \mathrm{s}$ on etched surfaces in the direction of ion incidence (or in the $x$-direction) continuously after the start of etching, as seen later in Figs. 6 and 7; thus, these snapshot images can be considered to be those which observers at rest on the traveling surface features would see every $T=W / v_{r} \approx$ $9 \mathrm{~s}$ and/or its multiples, where $W=50 \mathrm{~nm}$ is the length of one side of the square substrates being simulated.

Simulations exhibit that soon after the start of etching, the initially flat surfaces $(\mathrm{RMS}=0)$ are roughened randomly with small concavo-convex roughness features at any $\theta_{i}$ (stage I), while the evolution of surface features thereafter depends largely on $\theta_{i}{ }^{7,72,73}$ at intermediate $\theta_{i}=45^{\circ}$, small roughness features tend to merge with each other with time to form concavo-convex features enlarged laterally as well as vertically (stage II), which evolve further into pronounced ripple structures with wave crests/troughs elongated perpendicular to the direction of ion incidence (stage III). The stages I, II, and III correspond approximately to the respective time ranges in Fig. 2(d) exhibiting a little increase in RMS $(<0.3$, the low level of stochastic roughening) soon after the start of etching $\left(0<t<t_{1} \approx\right.$ a few seconds irrespective of $\left.r_{i}\right)$, the subsequent slight increase in RMS $(<0.5$, $\left.t_{1}<t<t_{2}\right)$, and then a significant increase in RMS $(<2)$ thereafter (a) $r_{\mathrm{i}}=1$

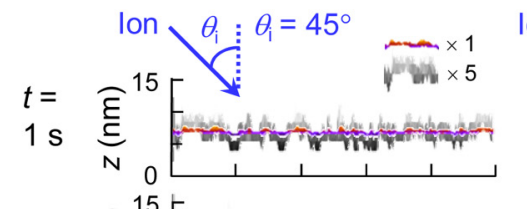

$3 s$

$12 \mathrm{~s}$

$21 \mathrm{~s}$

$30 \mathrm{~s}$

$39 \mathrm{~s}$

$48 \mathrm{~s}$

$66 \mathrm{~s}$

$93 \mathrm{~s}$

$120 \mathrm{~s}$
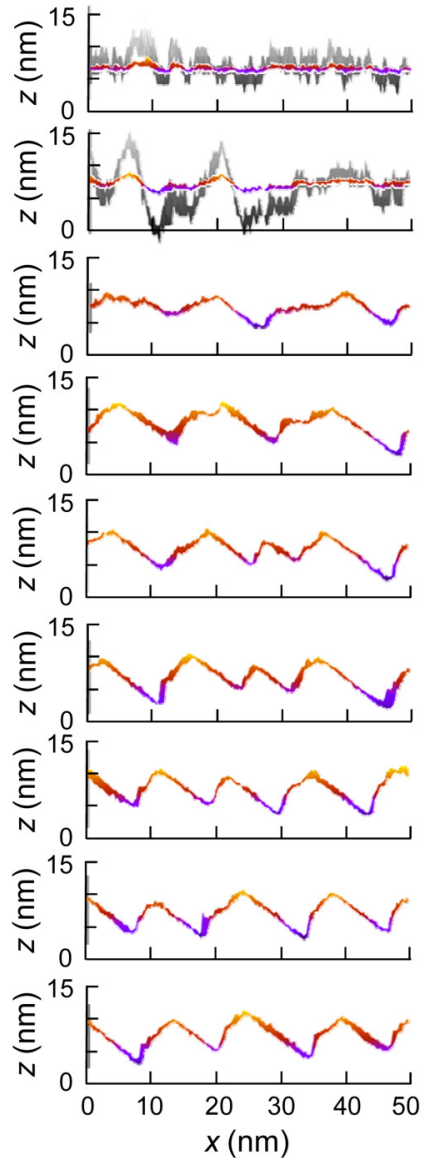

(b) $r_{\mathrm{i}}=0.9$
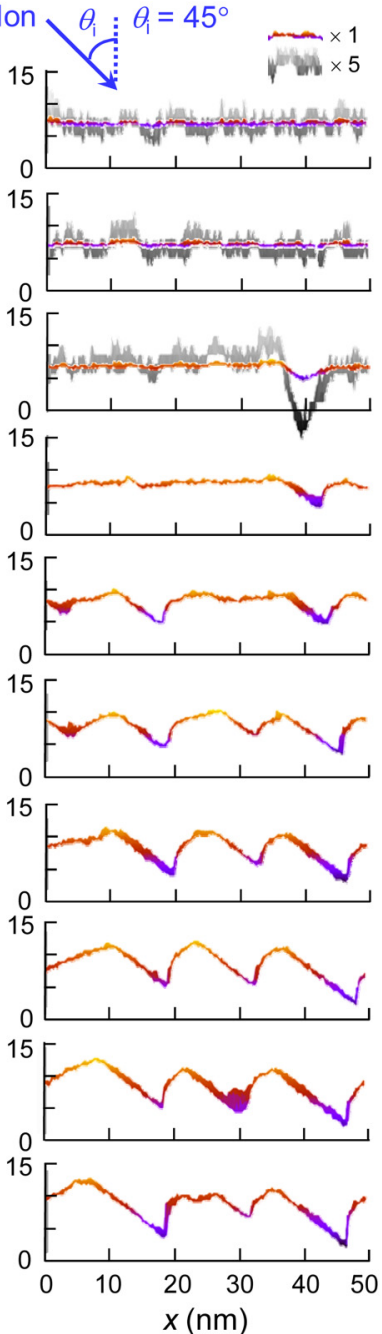

(c) $r_{\mathrm{i}}=0.85$
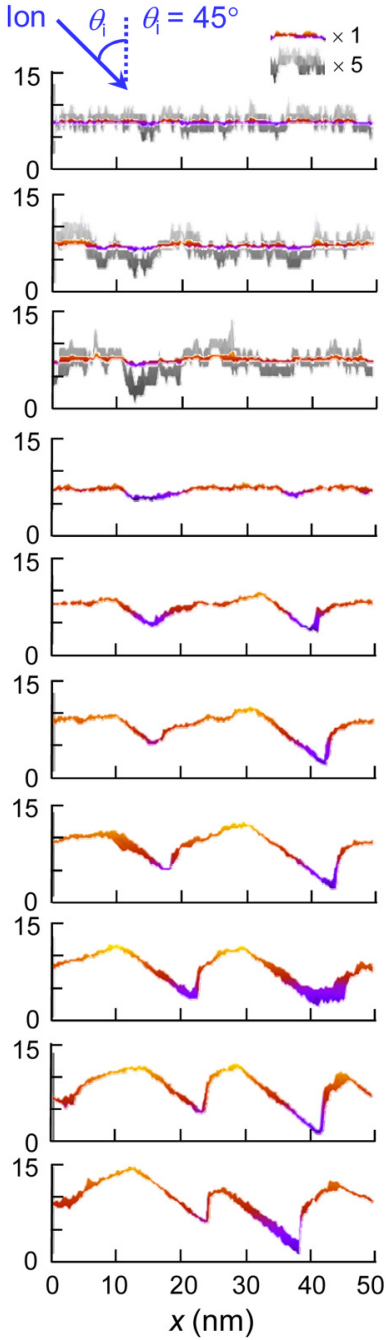

(d) $r_{\mathrm{i}}=0.8$
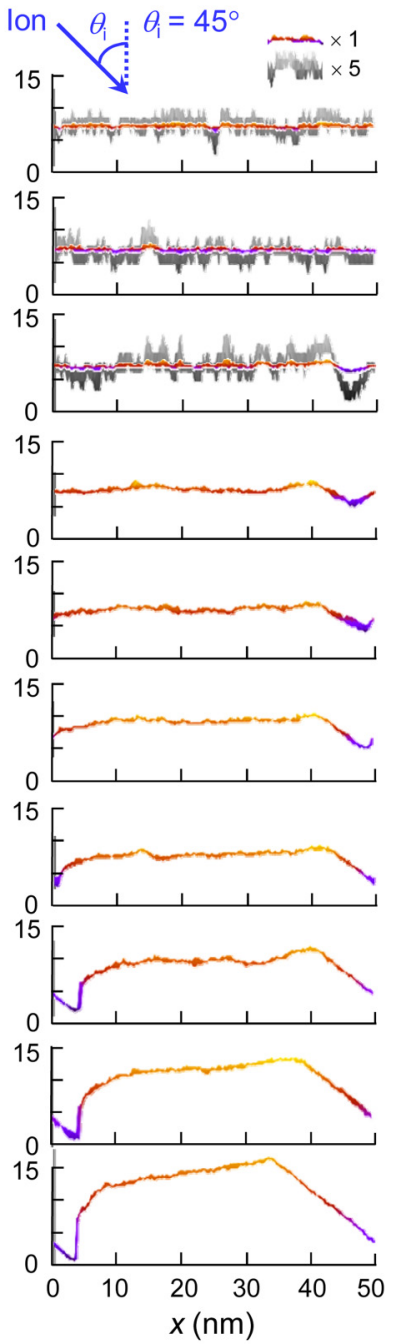

FIG. 5. Time evolution of representative side or cross-sectional views of surface features of Si during etching $(0 \leq t \leq 120 \mathrm{~s})$ for different $r_{i}=(\mathrm{a}) 1$, (b) 0.9 , (c) 0.85 , and (d) 0.8 with $E_{i}=100 \mathrm{eV}$ at $\theta_{i}=45^{\circ}$, corresponding to the preceding Fig. 4 . The line of sight here is perpendicular to the direction of ion incidence (or in the $y$-direction), and the simulation domain shown is $2 \mathrm{~nm}$ in width (in the $y$-direction at around the $x$ axis at $y=22,12,40$, and $40 \mathrm{~nm}$ for $r_{i}=1,0.9,0.85$, and 0.8 , respectively). 

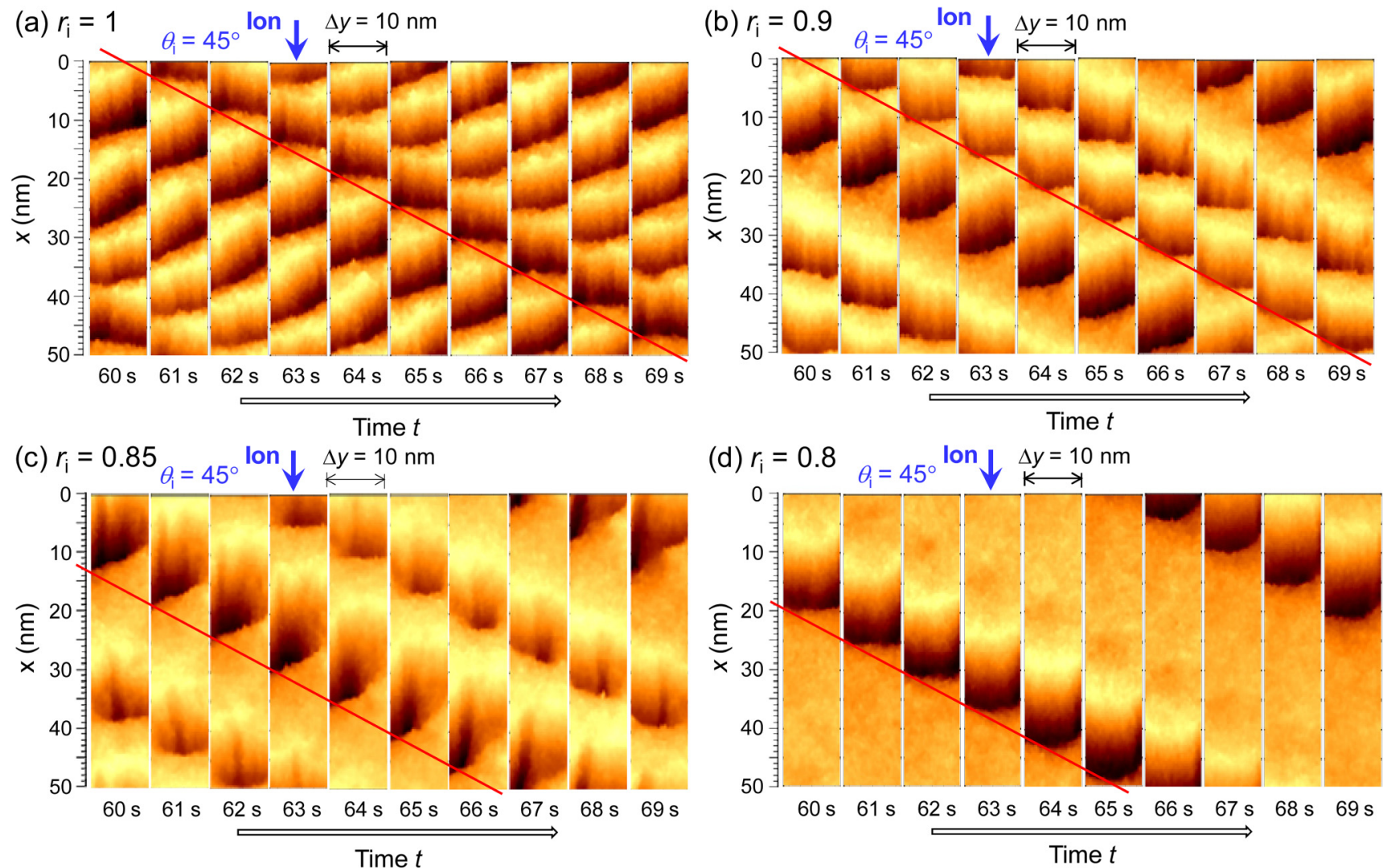

FIG. 6. Time sequence of surface features or ripple patterns of $\mathrm{Si}$ at $t=60-69 \mathrm{~s}$ during etching in $\mathrm{Cl}_{2}$ plasma for different ion reflection coefficients of $r_{i}=($ a) 1, (b) 0.9 , (c) 0.85 , and (d) 0.8 with $E_{i}=100 \mathrm{eV}$ at $\theta_{i}=45^{\circ}$ (top view, $\Delta x \times \Delta y=50 \times 10 \mathrm{~nm}^{2}$ of the total area $50 \times 50 \mathrm{~nm}^{2}$ at around the $x$ axis at $y=22,12,40$, and $40 \mathrm{~nm}$, respectively), extracted from simulations as in Fig. 1(b). The red straight lines in the respective figures are for guiding the eyes only, showing that given surface feature points move or travel laterally at a velocity $v_{r} \approx 5.5 \mathrm{~nm} / \mathrm{s}$ across the surfaces being etched in the direction of ion incidence (or in the $x$-direction).

$\left(t_{2}<t<t_{3}\right)$. The critical times concerned are longer for decreased $r_{i}$, being $t_{2} \sim 10,20,20$, and $30 \mathrm{~s}$ and $t_{3} \sim 35,60$, 90 , and $90 \mathrm{~s}$ for $r_{i}=1,0.9,0.85$, and 0.8 , respectively.

The evolution reaches quasi-steady state at around the end of stage III, exhibiting parallel-mode ripples characterized by approximately four, three, two, and one crest(s)/ trough(s) for $r_{i}=1,0.9,0.85$, and 0.8 , respectively; in other words, the distance between crests/troughs (crest-to-crest/ trough-to-trough) is larger, or the ripple wavelength is longer, for decreased $r_{i}$. For further decreased $r_{i}<0.8$ (or $r_{i} \leq r_{i}^{*} \approx 0.75$ ), the surfaces remain smooth at the low level of stochastic roughening during etching after only a little increase in roughness at the initial stage I, without the subsequent evolution of roughness and ripples. Note that the ASCeM-3D obeys periodic boundary conditions in horizontal $x$ - and $y$-directions (as mentioned in Sec. II), giving the surface heights/depths $z(x=0, y)=z(x=W, y) \quad$ and $z(x, y=0)=z(x, y=W)$ at boundaries; thus, in simulations, the number of wave crests/troughs formed should be an integer $(0,1,2,3,4, \ldots)$, or the ripple wavelength should be nominally a fraction $(\infty, 1,1 / 2,1 / 3,1 / 4, \ldots)$ of the simulation domain size $W=50 \mathrm{~nm}$. The smoothing/non-roughening for decreased $r_{i}<0.8$ at $\theta_{i}=45^{\circ}$ may be attributed partly to the wavelength of the ripples to be formed that would exceed the size limit $W$.

The top- and side-view images of Figs. 4 and 5 further exhibit that at stage II $\left(t_{1}<t<t_{2}\right)$, the roughness features enlarged at intervals are fewer in number density for decreased $r_{i}$; this in turn leads to the number of wave crests/ ripples elongated that is smaller (or to the ripple wavelength that is longer) for decreased $r_{i}$ at stage III $\left(t_{2}<t<t_{3}\right)$, when the roughness features enlarged are merged laterally (preferentially in the direction perpendicular to that of ion incidence) to form pronounced parallel-mode ripple structures. At stage III in side-view images of Fig. 5, the number of crests/troughs looks as if it increased (or the wavelength decreased) with time, owing to the lateral merging of roughness features, except the case $r_{i}=0.8$ where it remains one. Thus, we consider that the reduced ion reflection (or equivalently the enhanced smoothing) suppresses the formation of roughness features enlarged at intervals, which in turn leads to the decrease in number of wave crests/troughs elongated, or to the increase in wavelength of the ripples formed, for decreased $r_{i}$.

Figure 6 shows the time sequence of surface features or ripple patterns of $\mathrm{Si}$ at $t=60-69 \mathrm{~s}$ during etching in $\mathrm{Cl}_{2}$ plasma for different $r_{i}=1,0.9,0.85$, and 0.8 with $E_{i}=100 \mathrm{eV}$ at $\theta_{i}=45^{\circ}$ (top view, $\Delta x \times \Delta y=50 \times 10 \mathrm{~nm}^{2}$ of the total area $50 \times 50 \mathrm{~nm}^{2}$ at around the $x$ axis at $y=22,12,40$, and $40 \mathrm{~nm}$, respectively), extracted also from simulations as in Fig. 1(b). The corresponding representative side or cross-sectional views of surface features or ripple structures are shown in Fig. 7, where the figure properties (the line of sight and the simulation domain shown) are the same as in Fig. 5. A series 
(a) $r_{\mathrm{i}}=1$

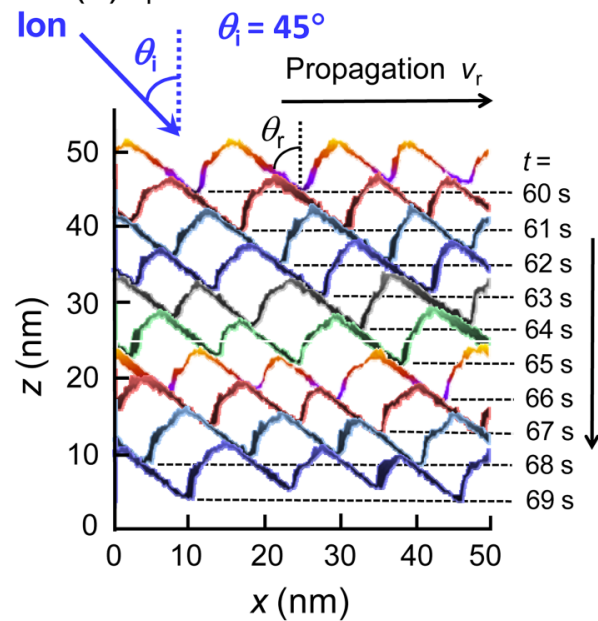

(c) $r_{\mathrm{i}}=0.85$

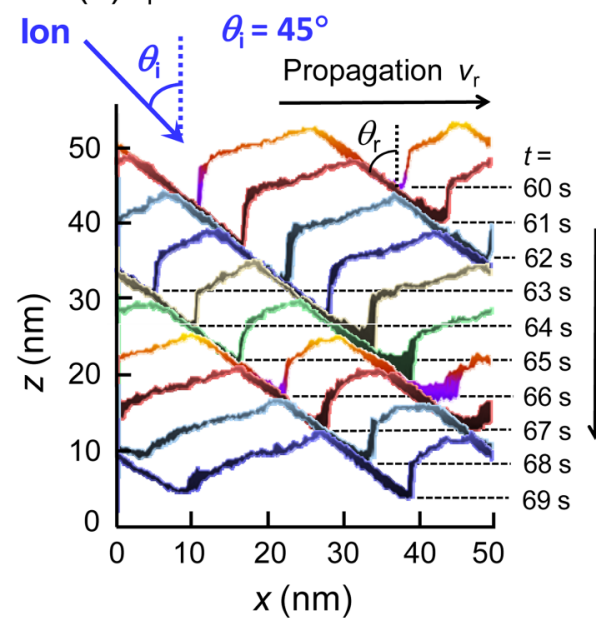

(b) $r_{\mathrm{i}}=0.9$

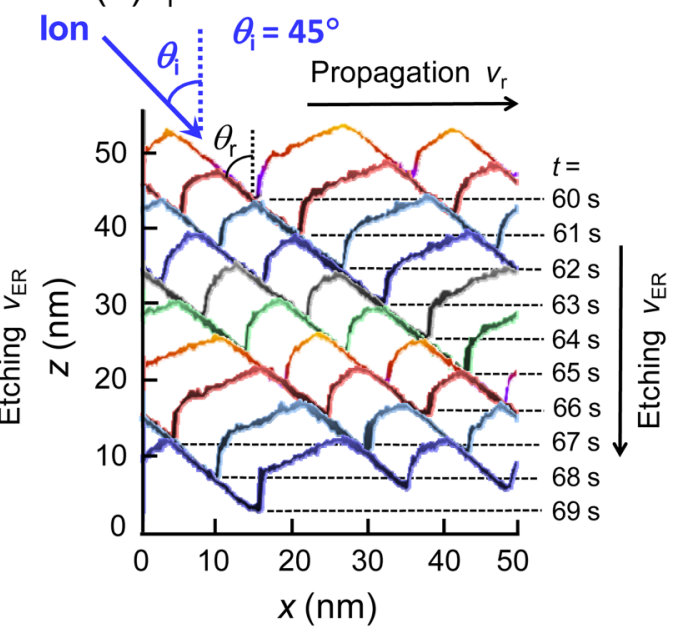

(d) $r_{\mathrm{i}}=0.8$

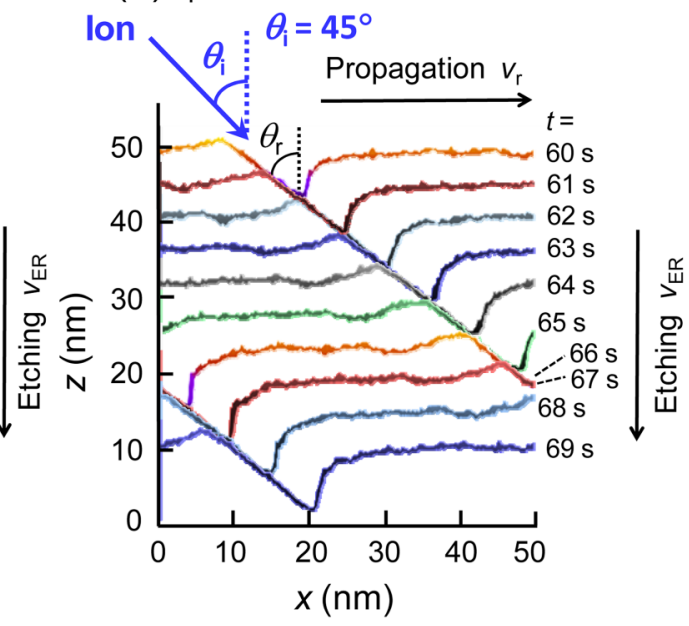

FIG. 7. Time sequence of representative side or cross-sectional views of surface features or ripple structures of Si at $t=60-69 \mathrm{~s}$ during etching for different $r_{i}$ $=$ (a) 1 , (b) 0.9 , (c) 0.85 , and (d) 0.8 with $E_{i}=100 \mathrm{eV}$ at $\theta_{i}=45^{\circ}$, corresponding to the preceding Fig. 6. The figure properties (the line of sight and the simulation domain shown) are the same as in Fig. 5. The surface features or ripples move or travel laterally at $v_{r} \approx 5.5 \mathrm{~nm} / \mathrm{s}$ across the surfaces being etched in the direction of ion incidence (or in the $x$-direction), with the surfaces being moved vertically downward (or in the negative $z$-direction) at $v_{\mathrm{ER}}=\mathrm{ER} / 60 \approx 4.3 \mathrm{~nm} / \mathrm{s}$ through etching; then, the ripple angle, defined as the angle between the downward slope of the ripple and the surface normal of substrates, is correlated with the ion incidence angle as approximately $\theta_{r} \approx \theta_{i}=45^{\circ}$.

of these time-varying top- and side-view images indicate that the surface features or ripples [characterized by four, three, two, and one wave crest(s)/trough(s) for $r_{i}=1,0.9,0.85$, and 0.8 , respectively] move or travel laterally across the surface being etched in the direction of ion incidence (or in the $x$-direction), with the surfaces being moved vertically downward (or in the negative $z$-direction) through etching. The propagation of parallel-mode ripples in the ion incidence direction has so far been observed in several IBS experiments $^{70,98-101}$ (although there is a recent exception), ${ }^{102}$ which is opposite to the predictions of continuum models ${ }^{71}$ and MC simulations ${ }^{97,103-106}$ of the ion beam-induced ripple formation. This discrepancy may suggest that the ion-induced ripple formation and evolution and its topography and dynamics are largely affected by the ion reflection followed by re-impingement on feature surfaces, the effects of which have not been included in IBS models and simulations, ${ }^{36,71,97}$ although they include surface processes such as ion-induced sputtering and surface diffusion of defects.
The average traveling velocity, derived from the time rate of change of the position of given surface feature points (e.g., those of wave crests/troughs), is approximately $v_{r} \approx 5.5 \mathrm{~nm} / \mathrm{s}$ almost independent of $r_{i}$, which is a little higher than the vertically downward $v_{\mathrm{ER}}=\mathrm{ER} / 60 \approx 4.3 \mathrm{~nm} / \mathrm{s}$ through etching; as a result, similar surface features or ripple patterns are seen to occur in cycles with a period of $T=W / v_{r} \approx 9 \mathrm{~s}$ at quasi-steady state in Figs. 4-7 [note that the images in Fig. 1(b) are those seen at $t=60 \mathrm{~s}$ between $t=48$ and $66 \mathrm{~s}$ in Fig. 4]. In simulations, the traveling velocity $v_{r}$ as well as the descending velocity $v_{\mathrm{ER}}$ was observed to remain almost unchanged during etching $(0<t<120 \mathrm{~s})$. Similar propagation velocities of parallel-mode ripples have been reported in IBS experiments as mentioned above using 30-keV Ga ${ }^{+}$beams: $^{70,98-101}$ typically (with $\Phi \approx 1 \times 10^{18}$ ions $\left./ \mathrm{cm}^{2}\right), v_{r} \approx 0.33 \mathrm{~nm} / \mathrm{s} \approx 44 \mathrm{~nm} /\left(10^{17} \mathrm{ions} \mathrm{cm}^{-2}\right)$ on $\mathrm{Si}$ at $\theta_{i}=30^{\circ}\left(\lambda_{r} \approx 100 \mathrm{~nm}\right) ;^{98} v_{r} \approx 0.75 v_{0}$ on $\mathrm{SiO}_{2}$ at $\theta_{i}=45^{\circ}$ $\left(\lambda_{r} \approx 200 \mathrm{~nm}, z_{r} \approx 5 \mathrm{~nm}, v_{0}\right.$ the erosion velocity $) ; 9 v_{r} \approx 2.3$ $\mathrm{nm} / \mathrm{s} \approx 66 \mathrm{~nm} /\left(10^{17}\right.$ ions cm $\left.{ }^{-2}\right)$ on $\mathrm{Cd}_{2} \mathrm{Nb}_{2} \mathrm{O}_{7}$ pyrochlore at 
$\theta_{i}=45^{\circ}\left(\lambda_{r} \approx 400 \mathrm{~nm}, z_{r} \approx 150 \mathrm{~nm}\right] ;{ }^{100}$ and $v_{r} \approx 77 \mathrm{~nm} /\left(10^{17}\right.$ ions $\left.\mathrm{cm}^{-2}\right)$ on $\mathrm{SiO}_{2}$ at $\theta_{i}=52^{\circ} \quad\left(\lambda_{r} \approx 450 \mathrm{~nm}, z_{r} \approx 130\right.$ $\mathrm{nm}) .^{70,101}$ Note that the present $v_{r} \approx 5.5 \mathrm{~nm} / \mathrm{s}$ corresponds to $\approx 1.3 v_{\mathrm{ER}}$ and to $\approx 55 \mathrm{~nm} /\left(10^{17}\right.$ ions $\left.\mathrm{cm}^{-2}\right)$.

The amplitude (crest-to-trough) as well as wavelength (crest-to-crest/trough-to-trough) of the ripples is derived from side-view images as in Figs. 5 and 7, indicating that at quasi-steady state, the amplitude is approximately in the range $z_{r} \approx 6-12 \mathrm{~nm}$ (increasing slightly with decreasing $r_{i}$ from 1 to 0.8 ). The increase of the amplitude $z_{r}$ with $r_{i}$ is not so significant as compared to that of the wavelength which is nominally in the range $\lambda_{r} \approx(1 / 4-1) W=12.5-50 \mathrm{~nm}$; in a sense, the tendency of $z_{r}$ reflects that of the rms roughness depending on $r_{i}$ [Figs. 2(b) and 3(b)]. It is further noted that at quasi-steady state, the ripple angle, defined as the angle between the downward slope of the ripple and the surface normal of substrates, is correlated with the ion incidence angle as approximately $\theta_{r} \approx \theta_{i}=45^{\circ}$ irrespective of $r_{i}$; in other words, the downward-sloping surfaces of the ripple are nearly parallel to the ion incidence, in such a way that no shadowing effects of the feature occur for incoming ions. ${ }^{37,48}$ On the other hand, the upward-sloping surfaces consist of lower parts having a steep slope followed by upper ones having a relatively gentle one, where the latter are more gently sloped for decreased $r_{i}$ with the ripple wavelength being increased. The steep slope of lower parts of the upward surfaces is attributed to faceting caused by re-impingement of ions reflected from the adjacent downward surfaces on incidence in addition to direct incidence of incoming ions, ${ }^{53}$ while the gentle slope of upper parts is to faceting caused only by incoming ions directly incident thereon.

For $r_{i}=1$, the slopes of upper and lower parts of the upward-sloping surfaces tend to be similar, approximately perpendicular to the ion incidence, exhibiting the formation of well-defined sawtooth-like ripples $\left(\lambda_{r} \approx 12.5 \mathrm{~nm}, z_{r} \approx 6\right.$ $\mathrm{nm}$ for $100-\mathrm{eV} \mathrm{Cl}^{+}$on $\mathrm{Si}$ at $\theta_{i}=45^{\circ}$ ) as observed in our plasma etching $^{78}$ as well as IBS $^{66-69,100,107-109}$ experiments: typically, $\lambda_{r}(\mathrm{~nm}) / z_{r}(\mathrm{~nm}) \approx 62 / 18$ for $0.5-\mathrm{keV} \mathrm{Cl}_{2}{ }^{+}$ (in practice, probably $\mathrm{SiCl}^{+}$as discussed earlier in Sec. III A) on $\mathrm{Si}$ at $\theta_{i} \approx 40^{\circ}, 78 \approx 350 / 55$ for $0.5-\mathrm{keV} \mathrm{Ar}^{+}$on $\mathrm{Si}$ at $70^{\circ},{ }^{66,67} \approx 60 / 5$ for $1.2-\mathrm{keV} \mathrm{Xe}{ }^{+}$on $\mathrm{Si}$ at $75^{\circ},{ }^{68} \approx 40 / 5$ for $2-\mathrm{keV} \mathrm{Kr}^{+}$on $\mathrm{Si}$ at $75^{\circ}, 69 \approx 400 / 150$ for $30-\mathrm{keV} \mathrm{Ga}^{+}$on $\mathrm{Cd}_{2} \mathrm{Nb}_{2} \mathrm{O}_{7}$ at $45^{\circ},{ }^{100} \approx 500 / 40$ for $20-\mathrm{keV} \mathrm{Ga}^{+}$on diamond at $80^{\circ},{ }^{107,108}$ and $\approx 250 / 50$ for $12-\mathrm{keV} \mathrm{N}^{+}$on $\mathrm{Si}$ at $60^{\circ} .^{109}$ Such characteristic sawtooth-like (or terrace-like) profiles with $\theta_{r} \approx \theta_{i}$ are assumed to reflect the limiting condition for the formation of parallel-mode ripple structures, ${ }^{37,48,78}$ which causes shadowing not to occur and faceting to be fully developed.

The wavelengths and amplitudes of fully developed sawtooth-like ripples with $\theta_{r} \approx \theta_{\mathrm{i}}$ for intermediate off-normal $\theta_{i}=40^{\circ}-60^{\circ}$, observed in the present simulations, plasma etching, ${ }^{78}$ and IBS experiments ${ }^{100,109}$ at different $E_{i}=0.1$ $-30 \mathrm{keV}$, appear to scale with the ion energy as $\lambda_{r} \sim E_{i}^{p}$ and $z_{r} \sim E_{i}^{q}$ with $p, q \approx 0.6 .^{78}$ The ripple wavelength and amplitude in IBS are appreciated to rely on ion energy, incidence angle, and fluence, ${ }^{36-39}$ and so this scaling may also suggest that the ion-induced formation and evolution of ripple structures is largely affected not only by the ion shadowing and faceting ${ }^{40,48}$ but also by the ion reflection followed by re-impingement on feature surfaces. ${ }^{53}$ In simulations, sawtooth-like profiles tend to be collapsed with decreasing $r_{i}$, where the reduced ion reflection suppresses the evolution of roughness features or ripple structures to both vertical and lateral extent; in effect, if sawtooth-like profiles were fully developed for decreased $r_{i}$, the roughness height/depth or the ripple amplitude would be more significantly increased, corresponding to the increase in wavelength.

Figure 8 shows the wavelength $\lambda_{r}$ and amplitude $z_{r}$ of parallel-mode ripples of $\mathrm{Si}$ as a function of ion reflection coefficient in the range $r_{i}=1-0.5$, together with the rms surface roughness [Figs. 2(b) and 2(d)], obtained through simulations of Si etching in $\mathrm{Cl}_{2}$ plasma with $E_{i}=100 \mathrm{eV}$ at $\theta_{i}=45^{\circ}$ as in Fig. 1(b). Also shown are the corresponding traveling velocity $v_{r}$ of the ripples, together with the etch rate or the surface descending velocity $v_{\mathrm{ER}}=\mathrm{ER} / 60$ [Figs. 2(a) and 2(c)]. These are the data at around $t=60 \mathrm{~s}$ after the start of etching, providing a summary of the ripple topography and dynamics at quasi-steady state depending on $r_{i}$, as seen in top- and side-view images of surface features and/or ripple structures of Figs. 4-7. Note that in simulations, the surface features were observed to move or travel laterally on etched surfaces in the direction of ion incidence during etching irrespective of $r_{i}=1-0$, although the roughness is substantially reduced and ripples fade away for decreased $r_{i}<0.8$. As the coefficient $r_{i}$ is decreased, the ripple wavelength $\lambda_{r}$ increases significantly, while the increase in amplitude $z_{r}$ with $r_{i}$ is not so significant as compared to that of the wavelength; as a result, sawtooth-like ripple profiles, pronounced for $r_{i}=1$, tend to be collapsed with decreasing $r_{i}$, although the ripples move or travel laterally across the surfaces being etched in the direction of ion incidence with the velocity $v_{r}$ almost independent of $r_{i}$. This is consistent with IBS studies showing that the enhanced smoothing leads to an increase in the wavelength of parallel-mode ripples at lower beam fluxes
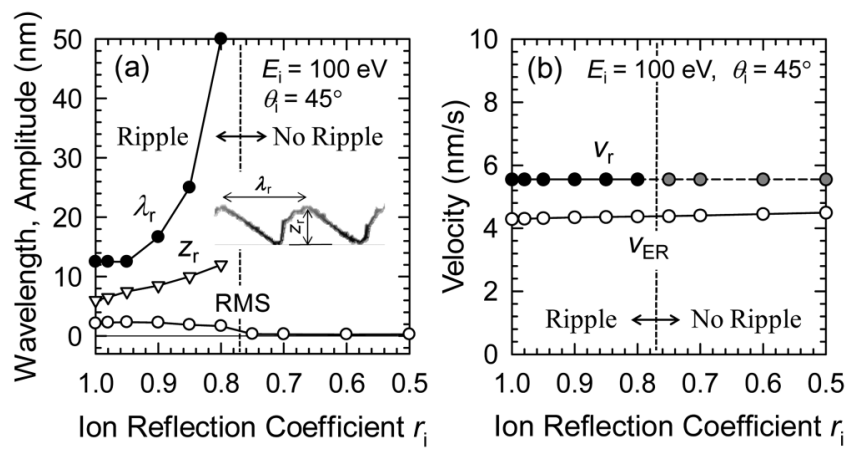

FIG. 8. (a) Wavelength $\lambda_{r}$ and amplitude $z_{r}$ of parallel-mode ripples of Si as a function of ion reflection coefficient in the range $r_{i}=1-0.5$, together with the rms surface roughness [Figs. 2(b) and 2(d)], obtained through simulations of Si etching in $\mathrm{Cl}_{2}$ plasma with $E_{i}=100 \mathrm{eV}$ at $\theta_{i}=45^{\circ}$ as in Fig. 1(b). Also shown are the corresponding (b) traveling velocity $v_{r}$ of the ripples, together with the etch rate or the surface descending velocity $v_{\mathrm{ER}}=\mathrm{ER} / 60$ [Figs. 2(a) and 2(c)]. The data shown are those at around $t=60 \mathrm{~s}$ after the start of etching, which are the averages extracted from several runs of the simulation except for nominal values of $\lambda_{r}$. These provide a summary of the ripple topography and dynamics at quasi-steady state depending on $r_{i}$, as seen in top- and side-view images of surface features and/or ripple structures of Figs. 4-7. 
and higher substrate temperatures. ${ }^{36,96,97}$ These results indicate that the reduced ion reflection affects significantly the ripple topography and dynamics induced by ion bombardment, which is equivalent to effects of the enhanced smoothing, although the smoothing in plasma etching is due to chemical effects of neutral reactants that are involved under shadowing.

\section{CONCLUSIONS}

We have investigated the origin of plasma-induced surface roughening and ripple formation, based on MC-based ASCeM-3D simulations of plasma-surface interactions and feature profile evolution during $\mathrm{Si}$ etching in Cl-based plasmas. The model took into account the $\mathrm{Cl}^{+}$ion reflection (and/or its penetration into substrates) through calculating the momentum and energy conservation in successive two-body elastic collisions with substrate $\mathrm{Si}$ atoms every ion incidence (not assuming specular reflection), thus giving internally the reflection probability $R_{i}\left(E_{i}, \theta\right)$ along with the distributions of reflected energies and angles. In this study, the "reflection coefficient $r_{i}$ " was further introduced in the model $\left(0 \leq r_{i} \leq 1\right)$, to quantitatively analyze the role and effects of ion reflection from microstructural feature surfaces on incidence. The coefficient $r_{i}$ represents the fraction of ions incident on surfaces with the reflection/penetration calculation scheme turned on, and so the $r_{i}$ is a measure of the reflection probability $\mathfrak{R}_{\mathrm{i}}$ for impacts of an ion species onto $\mathrm{Si}$ surfaces (a second condition of interest) relative to that $R_{i}$ for $\mathrm{Cl}^{+}$impacts (base condition): $\mathfrak{R}_{i}\left(E_{i}\right.$, $\theta)=r_{i} \times R_{i}\left(E_{i}, \theta\right)$, where $0 \leq r_{i}, R_{i}, \mathfrak{R}_{\mathrm{i}} \leq 1$, and $\theta$ is the local incidence angle on feature surfaces.

Simulations with ion incident energies in the range $E_{i}=20-500 \mathrm{eV}$ for incidence angles of $\theta_{i}=0^{\circ}, 45^{\circ}$, and $75^{\circ}$ onto substrate surfaces showed that as the coefficient $r_{i}$ is slightly decreased from unity, the roughness decreases substantially and the ripple formation fades away: the roughness remains at the low level of stochastic roughening $(\mathrm{RMS}<0.3$ $-0.5 \mathrm{~nm}$, no pronounced ripples at off-normal $\theta_{i}$ ) during etching for decreased $r_{i} \leq r_{i}^{*}$, where the critical $r_{i}^{*} \approx 0.95$, 0.75 , and 0.8 at $\theta_{i}=0^{\circ}, 45^{\circ}$, and $75^{\circ}$, respectively, e.g., for $E_{i}$ $=100 \mathrm{eV}$. This clearly indicates the crucial role of ion reflection from feature surfaces on incidence at microscale during plasma etching: it is indispensable in plasma-induced surface roughening and rippling, and their degree relies significantly on the reflectivity of ions. The decrease in roughness (or smoothing/non-roughening) with reduced ion reflection tends to be less significant at increased $E_{i}$ and $\theta_{i}$ : the higher the $E_{i}$ and the $\theta_{i}$, the smaller is the critical $r_{i}^{*}(\approx 0.95-0.75)$ or the larger is the deviation of the critical $r_{i}^{*}$ from unity.

The critical value $r_{i}^{*} \approx 0.95-0.8$ at normal $\theta_{i}=0^{\circ}$ is consistent with our experiments of ICP plasma etching of Si in $\mathrm{Cl}_{2}$ exhibiting the roughening-smoothing transition at increased $E_{i}>200-300 \mathrm{eV},{ }^{7,74,75}$ where the predominant ion flux was found to change from reactive $\mathrm{Cl}_{2}{ }^{+} / \mathrm{SiCl}_{3}{ }^{+}$(base condition) to depositive $\mathrm{SiCl}^{+}$(a second condition); in effect, the reflection probabilities tend to be lower for the latter than for the former, according to our MD simulations. . $^{77}$ Moreover, the smaller $r_{i}^{*} \approx 0.8-0.75$ at off-normal $\theta_{i}=45^{\circ}$ and $75^{\circ}$ (as compared to $r_{i}^{*} \approx 0.95-0.8$ at $\theta_{i}=0^{\circ}$ ) is also consistent with our experiments of $\mathrm{Si}$ etching in $\mathrm{Cl}_{2}$ plasmas using sheath control plates, ${ }^{78}$ demonstrating the ripple formation under conditions of $E_{i} \approx 500 \mathrm{eV}$ with $\theta_{i} \approx 40^{\circ}$ and $80^{\circ}$ (though in the smoothing mode for $\theta_{i}=0^{\circ}$, where the predominant ions were $\mathrm{SiCl}^{+}$with relatively low reflectivity). Similarity of the smoothing/non-roughening behavior between the reduced ion reflection and the increased reactant flux ${ }^{73}$ and surface temperature ${ }^{92}$ in simulations gives an insight into the reason why the plasma-induced formation and evolution of roughness and ripples is highly sensitive to the degree of ion reflection: small deviations of the critical $r_{i}^{*}$ from unity are ascribed to chemical or smoothing effects of neutral reactants incident preferentially on top of roughness features under the effects of shadowing, which compete with the roughening and rippling caused by ion bombardment.

There would be some other experimental observations of plasma-induced surface roughening and rippling interpreted similarly according to the results of this study, where the understanding of plasma conditions is required, including the predominant ion species and their reflection probabilities on surfaces concerned. In any case, the cause-and-effect relationships between the ion reflection and the surface roughness in plasma etching have not been fully understood due to their complexity; in effect, the former crucially affects the latter as in this study, while in turn, the former (reflection probability, distributions of reflected energies and angles) is affected by the latter as in earlier MD studies of Helmer and Graves. ${ }^{110}$

Simulations further showed that at intermediate offnormal $\theta_{i}=45^{\circ}$, the ripple wavelength increases significantly from $\lambda_{r} \approx 12.5$ to $50 \mathrm{~nm}$ as the coefficient is decreased from $r_{i}=1$ to 0.8 for a given $E_{i}=100 \mathrm{eV}$, while the increase in amplitude with $r_{i}$ is not so significant as compared to that of the wavelength; as a result, sawtooth-like ripple profiles pronounced for $r_{i}=1$ tend to be collapsed with decreasing $r_{i}$, although the ripples move or travel laterally across the surfaces being etched in the direction of ion incidence with the velocity $v_{r} \approx 5.5 \mathrm{~nm} / \mathrm{s}$ almost independent of $r_{i}$. This is consistent with IBS studies showing that the enhanced smoothing leads to an increase in the wavelength of parallel-mode ripples at lower beam fluxes and higher substrate temperatures; ${ }^{36,96,97}$ moreover, the ripple propagation is also consistent with experimental observations during IBS, ${ }^{70,98-101}$ and sawtooth-like (or terrace-like) ripple profiles are with observations in plasma etching ${ }^{78}$ and IBS $^{66-69,100,107-109}$ experiments. Thus, we consider that the ripple topography and dynamics induced by ion bombardment are also significantly affected by the ion reflection followed by re-impingement on feature surfaces, where the reduced ion reflection is effectively equivalent to the enhanced smoothing, although the smoothing in plasma etching is caused by chemical effects of neutral reactants that are involved under shadowing.

The results and understandings obtained in this study would be intriguing and informative for ion beam- as well as plasma-surface interactions, providing insight into the mechanisms underlying a number of experimental observations of ion-induced surface morphology evolution. Modeling and simulation studies like the present one, taking into account self-consistently the effects of ion reflection, should be useful 
and indispensable to unravel the complex cause-and-effect relationships between it and surface roughness, and to quantitatively understand what plasma and/or beam conditions lead to what degree of surface roughening and rippling.

\section{ACKNOWLEDGMENTS}

This work was supported by Grants-in-Aid for Scientific Research (Grant Nos. 21110008 and 15H03582) from MEXT and JSPS, Japan. One of the authors (K.O.) gratefully thanks Professor Y. Setsuhara (Osaka University) for kind support throughout preparing this article.

${ }^{1}$ C. G. N. Lee, K. J. Kanarik, and R. A. Gottscho, J. Phys. D: Appl. Phys. 47, 273001 (2014).

${ }^{2}$ K. J. Kanarik, T. Lill, E. A. Hudson, S. Sriraman, S. Tan, J. Marks, V. Vahedi, and R. A. Gottscho, J. Vac. Sci. Technol. A 33, 020802 (2015).

${ }^{3}$ K. Patel, T.-J. King Liu, and C. J. Spanos, IEEE Trans. Electron Devices 56, 3055 (2009).

${ }^{4}$ X. Sun and T.-J. King Liu, IEEE Trans. Semicond. Manuf. 23, 311 (2010).

${ }^{5}$ W. Guo and H. H. Sawin, J. Phys. D: Appl. Phys. 42, 194014 (2009), and references therein.

${ }^{6}$ G. S. Oehrlein, R. J. Phaneuf, and D. B. Graves, J. Vac. Sci. Technol. A 29, 010801 (2011), and references therein.

${ }^{7}$ K. Ono, N. Nakazaki, H. Tsuda, Y. Takao, and K. Eriguchi, J. Phys. D: Appl. Phys. 50, 414001 (2017), and references therein.

${ }^{8}$ D. L. Goldfarb, A. P. Mahorowala, G. M. Gallatin, K. E. Petrillo, K. Temple, M. Angelopoulos, S. Rasgon, H. H. Sawin, S. D. Allen, M. C. Lawason, and R. W. Kwong, J. Vac. Sci. Technol. B 22, 647 (2004).

${ }^{9}$ J. Thiault, J. Foucher, J. H. Tortai, O. Joubert, S. Landis, and S. Pauliac, J. Vac. Sci. Technol. B 23, 3075 (2005).

${ }^{10}$ E. Gogolides, V. Constantoudis, G. P. Patsis, and A. Tserepi, Microelectron. Eng. 83, 1067 (2006).

${ }^{11}$ E. Pargon, M. Martin, J. Thiault, O. Joubert, J. Foucher, and T. Lill, J. Vac. Sci. Technol. B 26, 1011 (2008).

${ }^{12}$ G. Kokkoris, V. Constantoudis, and E. Gogolides, IEEE Trans. Plasma Sci. 37, 1705 (2009).

${ }^{13}$ A. Hiraiwa and A. Nishida, J. Appl. Phys. 106, 074905 (2009); J. Vac. Sci. Technol. B 28, 1132 (2010); Jpn. J. Appl. Phys. 50, 086502 (2011).

${ }^{14}$ R. L. Bruce, F. Weilnboeck, T. Lin, R. J. Phaneuf, G. S. Oehrlein, B. K. Long, C. G. Wilson, and A. Alizadeh, J. Vac. Sci. Technol. B 29, 041604 (2011).

${ }^{15}$ L. Azarnouche, E. Pargon, K. Menguelti, M. Fouchier, O. Joubert, P. Gouraud, and C. Verove, J. Vac. Sci. Technol. B 31, 012205 (2013).

${ }^{16} \mathrm{~N}$. Vourdas, D. Kontziampasis, G. Kokkoris, V. Constantoudis, A. Goodyear, A. Tserepi, M. Cooke, and E. Gogolides, Nanotechnology 21, 085302 (2010).

${ }^{17}$ G. Kokkoris, Eur. Phys. J. Appl. Phys. 56, 24012 (2011).

${ }^{18}$ E. Gogolides, V. Constantoudis, G. Kokkoris, D. Kontziampasis, K. Tsougeni, G. Boulousis, M. Vlachopoulou, and A. Tserepi, J. Phys. D: Appl. Phys. 44, 174021 (2011).

${ }^{19}$ G. Kokkoris and E. Gogolides, J. Phys. D: Appl. Phys. 45, 165204 (2012).

${ }^{20}$ M.-E. Vlachopoulou, G. Kokkoris, C. Cardinaud, E. Gogolides, and E. Tserepi, Plasma Process. Polym. 10, 29 (2013).

${ }^{21}$ J. Bae and I. J. Lee, Sci. Rep. 5, 10126 (2015).

${ }^{22}$ P. Brault, P. Dumas, and F. Salvan, J. Phys. Condens. Matter 10, L27 (1998).

${ }^{23}$ Y.-P. Zhao, J. T. Drotar, G.-C. Wang, and T.-M. Lu, Phys. Rev. Lett. 82, 4882 (1999).

${ }^{24}$ J. T. Drotar, Y.-P. Zhao, T.-M. Lu, and G.-C. Wang, Phys. Rev. B 61, 3012 (2000); ibid. 62, 2118 (2000).

${ }^{25}$ Y. Yin and H. H. Sawin, J. Vac. Sci. Technol. A 26, 151 (2008).

${ }^{26}$ E. Zakka, V. Constantoudis, and E. Gogolides, IEEE Trans. Plasma Sci. 35, 1359 (2007).

${ }^{27}$ G. Kokkoris, V. Constantoudis, P. Angelikopoulos, G. Boulousis, and E. Gogolides, Phys. Rev. B 76, 193405 (2007).
${ }^{28}$ O. Mourey, C. Petit-Etienne, G. Cunge, M. Darnon, E. Despiau-Pujo, P. Brichon, E. Lattu-Romain, M. Pons, and O. Joubert, J. Vac. Sci. Technol. A 34, 041306 (2016).

${ }^{29}$ S. Mouchtouris and G. Kokkoris, Plasma Process. Polym. 13, 147 (2016).

${ }^{30}$ Y. Zhang, C. Huard, S. Sriaman, J. Belen, A. Paterson, and M. J. Kushner, J. Vac. Sci. Technol. A 35, 021303 (2017).

${ }^{31}$ Y. Yin, S. Rasgon, and H. H. Sawin, J. Vac. Sci. Technol. B 24, 2360 (2006).

${ }^{32}$ Y. Yin and H. H. Sawin, J. Vac. Sci. Technol. A 25, 802 (2007).

${ }^{33}$ W. Guo and H. H. Sawin, J. Vac. Sci. Technol. A 28, 259 (2010).

${ }^{34}$ G. Carter, J. Phys. D: Appl. Phys. 34, R1 (2001), and references therein.

${ }^{35}$ M. A. Makeev, R. Cuerno, and A.-L. Barabási, Nucl. Instrum. Methods B 197, 185 (2002), and references therein.

${ }^{36}$ W. L. Chan and E. Chason, J. Appl. Phys. 101, 121301 (2007), and references therein.

${ }^{37}$ T. K. Chini, D. P. Datta, and S. R. Bhattacharyya, J. Phys.: Condens. Matter 21, 224004 (2009), and references therein.

${ }^{38}$ A. Keller and S. Facsko, Materials 3, 4811 (2010), and references therein.

${ }^{39}$ J. Munõz-Garcí, L. Vázquez, M. Castro, R. Gago, A. Redondo-Cubero, A. Moreno-Barrado, and R. Cuerno, Mater. Sci. Eng. R 86, 1 (2014), and references therein.

${ }^{40}$ R. M. Bradley and J. M. E. Harper, J. Vac. Sci. Technol. A 6, 2390 (1988).

${ }^{41}$ R. Cureno and A.-L. Barabási, Phys. Rev. Lett. 74, 4746 (1995).

${ }^{42}$ R. Cuerno, H. A. Makse, S. Tomassone, S. T. Harrington, and H. E. Stanley, Phys. Rev. Lett. 75, 4464 (1995).

${ }^{43}$ S. Park, B. Kahng, H. Jeong, and A.-L. Barabási, Phys. Rev. Lett. 83, 3486 (1999).

${ }^{44}$ E. Chason, T. M. Mayer, B. K. Kellerman, D. T. Mcllroy, and A. J. Howard, Phys. Rev. Lett. 19, 3040 (1994).

${ }^{45}$ T. M. Mayer, E. Chason, and A. J. Howard, J. Appl. Phys. 76, 1633 (1994).

${ }^{46}$ M. A. Makeev and A.-L. Barabási, Appl. Phys. Lett. 71, 2008 (1997).

${ }^{47}$ C. C. Umbach, R. L. Headrick, and K.-C. Chang, Phys. Rev. Lett. 87, 246104 (2001).

${ }^{48}$ G. Carter, J. Appl. Phys. 85, 455 (1999).

${ }^{49}$ J. Muñoz-García, M. Castro, and R. Cuerno, Phys. Rev. Lett. 96, 086101 (2006).

${ }^{50}$ B. Davidovitch, M. J. Aziz, and M. P. Brenner, Phys. Rev. B 76, 205420 (2007).

${ }^{51}$ C. S. Madi, E. Anzenberg, K. F. Ludwig, Jr, and M. J. Aziz, Phys. Rev. Lett. 106, 066101 (2011).

${ }^{52}$ O. Bobes, K. Zhang, and H. Hofsäss, Phys. Rev. B 86, 235414 (2012).

${ }^{53}$ W. Hauffe, Phys. Stat. Sol. (a) 35, K93 (1976).

${ }^{54}$ H. Hansen, A. Redinger, S. Messlinger, G. Stoian, Y. Rosandi, H. M. Urbassek, U. Linke, and T. Michely, Phys. Rev. B 73, 235414 (2006).

${ }^{55}$ M. Z. Hossain, K. Das, J. B. Freund, and H. T. Johnson, Appl. Phys. Lett. 99, 151913 (2011).

${ }^{56}$ Y. Rosandi and H. M. Urbassek, Phys. Rev. B 85, 155430 (2012).

${ }^{57}$ R. J. Hoekstra, M. J. Kushner, V. Sukharev, and P. Schoenborn, J. Vac. Sci. Technol. B 16, 2102 (1998).

${ }^{58}$ S. Abdollahi-Alibeik, J. P. McVittie, K. C. Saraswat, V. Sukharev, and P. Schoenborn, J. Vac. Sci. Technol. A 17, 2485 (1999).

${ }^{59}$ M. A. Vyvoda, M. Li, D. B. Graves, H. Lee, M. V. Malyshev, F. P. Klemens, J. C. T. Lee, and V. M. Donnelly, J. Vac. Sci. Technol. B. 18, 820 (2000).

${ }^{60}$ A. P. Mahorowala and H. H. Sawin, J. Vac. Sci. Technol. B 20, 1064 (2002), ibid. 20, 1077 (2002).

${ }^{61}$ W. Jin, S. A. Vitae, and H. H. Sawin, J. Vac. Sci. Technol. A 20, 2106 (2002).

${ }^{62}$ W. Jin and H. H. Sawin, J. Vac. Sci. Technol. A 21, 911 (2003).

${ }^{63}$ Y. Osano, M. Mori, N. Itabashi, K. Takahashi, K. Eriguchi, and K. Ono, Jpn. J. Appl. Phys. 45, 8157 (2006).

${ }^{64}$ K. Ono, H. Ohta, and K. Eriguchi, Thin Solid Films 518, 3461 (2010).

${ }^{65}$ H. Tsuda, M. Mori, Y. Takao, K. Eriguchi, and K. Ono, Thin Solid Films 518, 3475 (2010); Jpn. J. Appl. Phys. 49, 08JE01 (2010).

${ }^{66}$ T. Basu, J. R. Mohanty, and T. Som, Appl. Surf. Sci. 258, 9944 (2012).

${ }^{67}$ T. Basu, D. P. Datta, and T. Som, Nanoscale Res. Lett. 8, 289 (2013).

${ }^{68} \mathrm{M}$. Teichmann, J. Lorbeer, F. Frost, and B. Rauschenbach, Nanoscale Res. Lett. 9, 439 (2014).

${ }^{69}$ M. Engler, S. Macko, F. Frost, and T. Michely, Phys. Rev. B 89, 245412 (2014).

${ }^{70}$ H. Gnaser, B. Reuscher, and A. Zeuner, Nucl. Instrum. Methods B $\mathbf{2 8 5}$, $142(2012)$ 
${ }^{71}$ D. A. Pearson and R. M. Bradley, J. Phys.: Condens. Matter 27, 015010 (2015).

${ }^{72}$ H. Tsuda, Y. Takao, K. Eriguchi, and K. Ono, Jpn. J. Appl. Phys. 51, 08HC01 (2012).

${ }^{73}$ H. Tsuda, N. Nakazaki, Y. Takao, K. Eriguchi, and K. Ono, J. Vac. Sci. Technol. B 32, 031212 (2014).

${ }^{74}$ N. Nakazaki, H. Tsuda, Y. Takao, K. Eriguchi, and K. Ono, J. Appl. Phys. 116, 223302 (2014).

${ }^{75}$ N. Nakazaki, H. Matsumoto, H. Tsuda, Y. Takao, K. Eriguchi, and K. Ono, Appl. Phys. Lett. 109, 204101 (2016).

${ }^{76}$ N. Nakazaki, Y. Takao, K. Eriguchi, and K. Ono, Jpn. J. Appl. Phys. 53, 056201 (2014); J. Appl. Phys. 118, 233304 (2015).

${ }^{77}$ N. Nakazaki, Y. Takao, K. Eriguchi, and K. Ono, "Molecular dynamics simulations of oblique incidence of silicon chloride ions during $\mathrm{Si}$ etching in Cl-based plasmas," Jpn. J. Appl. Phys. (unpublished).

${ }^{78}$ N. Nakazaki, H. Matsumoto, S. Sonobe, T. Hatsuse, H. Tsuda, Y. Takao, K. Eriguchi, and K. Ono, AIP Adv. 8, 055027 (2018).

${ }^{79}$ C. Steinbrüchel, Appl. Phys. Lett. 55, 1960 (1989).

${ }^{80} \mathrm{P}$. Sigmund, in Sputtering by Particle Bombardment I. Physical Sputtering of Single-Element Solids, edited by R. Behrisch (Springer, Berlin, 1981), Chap. 2, pp. 9-71.

${ }^{81}$ J. P. Biersack and W. Eckstein, Appl. Phys. A 34, 73 (1984).

${ }^{82}$ J. F. Ziegler, J. P. Biersack, and U. Littmark, The Stopping and Range of Ions in Solids (Pergamon, New York, 1985), pp. 48-49.

${ }^{83}$ J. F. Ziegler, J. P. Biersack, and M. D. Ziegler, SRIM - The Stopping and Range of Ions in Matter (SRIM Co., Chester, MD, 2008), pp. 2-31-32.

${ }^{84}$ E. A. Ogryzlo, D. E. Ibbotson, D. L. Flamm, and J. A. Mucha, J. Appl. Phys. 67, 3115 (1990).

${ }^{85}$ W. Guo, B. Bai, and H. H. Sawin, J. Vac. Sci. Technol. A 27, 388 (2009).

${ }^{86}$ W. Guo and H. H. Sawin, J. Vac. Sci. Technol. A 27, 1326 (2009).

${ }^{87}$ K.-T. Sung and S. W. Pang, Jpn. J. Appl. Phys., Part 1 33, 7112 (1994).

${ }^{88}$ W. S. Hwang, B.-J. Cho, D. S. H. Chan, S. W. Lee, and W. J. Yoo, J. Electrochem. Soc. 155, H6 (2008).

${ }^{89}$ R. Pétri, P. Brault, O. Vatel, D. Henry, E. André, P. Dumas, and F. Salvan, J. Appl. Phys. 75, 7498 (1994).

${ }^{90}$ M. Martin and G. Cunge, J. Vac. Sci. Technol. B 26, 1281 (2008).
${ }^{91}$ S. Tinck, W. Boullart, and A. Bogaerts, Plasma Sources Sci. Technol. 20, 045012 (2011).

${ }^{92}$ T. Hatsuse, Master's thesis, Kyoto University, 2015.

${ }^{93}$ M. Dalvie, R. T. Farouki, and S. Hamaguchi, IEEE Trans. Electron Devices 39, 1090 (1992).

${ }^{94}$ G. Kokkoris, A. G. Boudouvis, and E. Gogolides, J. Vac. Sci. Technol. A 24, 2008 (2006).

${ }^{95}$ H. Fukumoto, K. Eriguchi, and K. Ono, Jpn. J. Appl. Phys. 48, 096001 (2009).

${ }^{96}$ W. L. Chan and E. Chason, Phys. Rev. B 72, 165418 (2005).

${ }^{97}$ E. Chason and W. L. Chan, J. Phys.: Condens. Matter 21, 224016 (2009).

${ }^{98}$ S. Habenicht, K. P. Lieb, J. Koch, and A. D. Wieck, Phys. Rev. B 65 , 115327 (2002).

${ }^{99}$ P. F. A. Alkemade, Phys. Rev. Lett. 96, 107602 (2006).

${ }^{100}$ Q. Wei, J. Lian, L. A. Boatner, L. M. Wang, and R. C. Ewing, Phys. Rev. B 80, 085413 (2009).

${ }^{101}$ D. Kramczynski, B. Reuscher, and H. Gnaser, Phys. Rev. B 89, 205422 (2014).

${ }^{102}$ H. Hofsäss, K. Zhang, H. G. Gehrke, and C. Brüsewitz, Phys. Rev. B 88, 075426 (2013).

${ }^{103}$ I. Koponen, M. Hautala, and O.-P. Sievänen, Phys. Rev. Lett. 78, 2612 (1997).

${ }^{104}$ A. K. Hartmann, R. Kree, U. Geyer, and M. Kölbel, Phys. Rev. B 65, 193403 (2002)

${ }^{105}$ E. O. Yewande, A. K. Hartmann, and R. Kree, Phys. Rev. B 71, 195405 (2005).

${ }^{106}$ E. Chason, W. L. Chan, and M. S. Bharathi, Phys. Rev. B 74, 224103 (2006).

${ }^{107}$ D. P. Adams, M. J. Vasile, T. M. Mayer, and V. C. Hodges, J. Vac. Sci. Technol. B 21, 2334 (2003).

${ }^{108}$ D. P. Adams, T. M. Mayer, M. J. Vasile, and K. Archuleta, Appl. Surf. Sci. 252, 2432 (2006).

${ }^{109}$ P. Karmakar and B. Satpati, J. Appl. Phys. 120, 025310 (2016).

${ }^{110}$ B. A. Helmer and D. B. Graves, J. Vac. Sci. Technol. A 15, 2252 (1997); ibid. 16, 3502 (1998); ibid. 17, 2759 (1999). 